Alma Mater Studiorum - Università di Bologna DEPARTMENT OF ECONOMICS

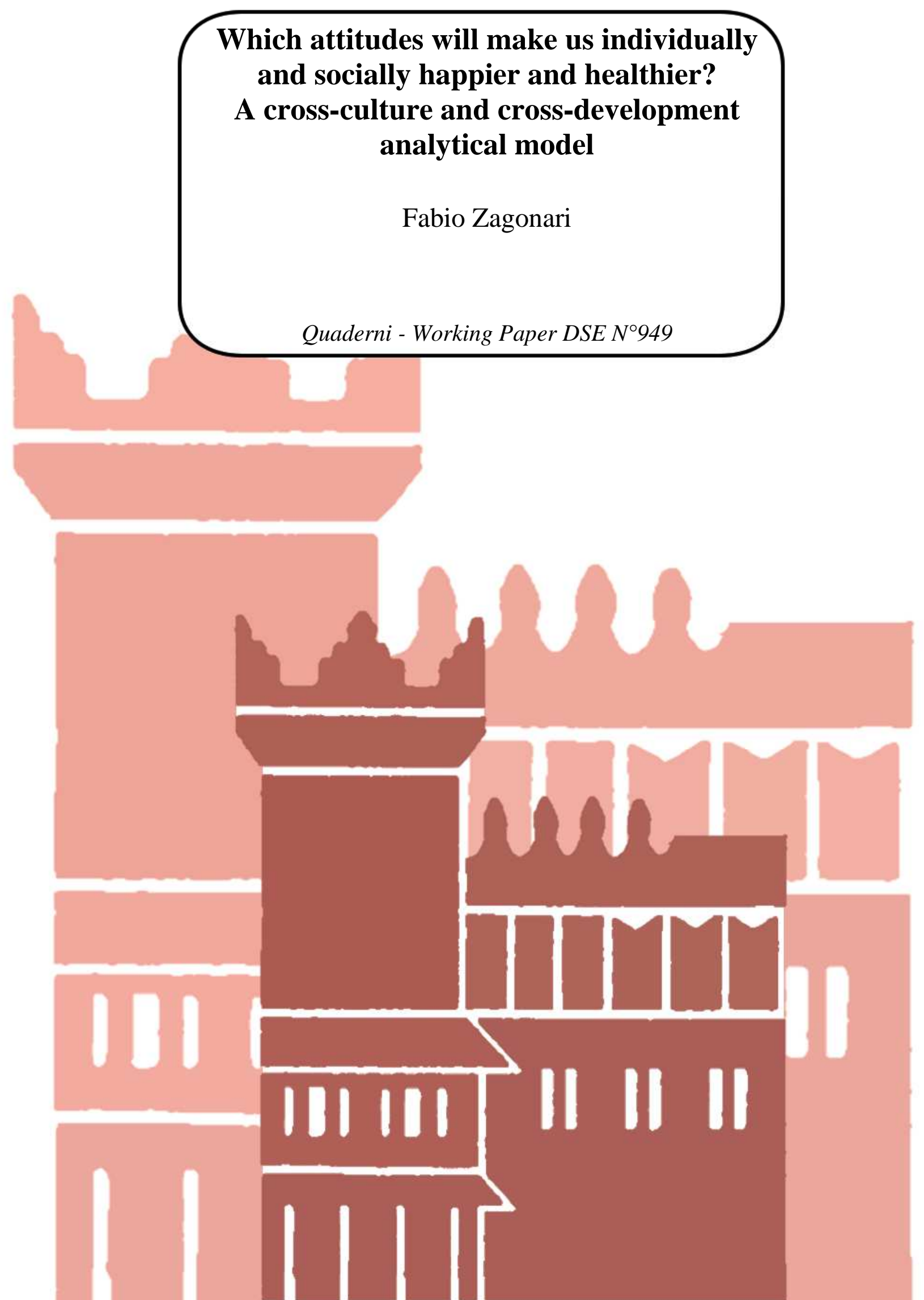




\title{
Which attitudes will make us individually and socially happier and healthier? A cross-culture and cross-development analytical model
}

\author{
Fabio Zagonari \\ Dipartimento di Scienze Economiche, Università di Bologna, via Angherà 22, 47921 Rimini (Italy) \\ Phone: 00390541 434135, Fax: 00390541 434120, E-mail: fabio.zagonari@unibo.it
}

June 2014

\begin{abstract}
This paper describes a dynamic system for the interrelationships between happiness and health that considers three main attitudes to life: $\alpha, \beta$, and $\gamma$ for Aristotelian, Epicurean, and Stoic, respectively. All variables that have been shown by empirical and theoretical studies to affect individual health and happiness are included (i.e., employment, occupation, education, ethical freedom, equity in achievements). Three main approaches are considered: behavioural and statistical ex-ante, and expost behavioural. I develop the model to rank the three attitudes in terms of health for a given happiness level, and consequently, provide insights into which attitude should be adopted by each individual, according to their characteristics: individuals in Protestant and non-Protestant Christian societies should adopt $\beta$ and $\gamma$ attitudes, respectively; educated individuals should adopt a $\gamma$ attitude; and poor individuals should adopt an $\alpha$ attitude. Based on this analysis, I provide insights into which attitude actually is adopted by each society by comparing predicted health and achievement levels with the observed life expectancy at birth and per capita gross domestic product levels in 107 countries, thus providing an empirical test of the analytical model. This analysis revealed a prevalence of $\beta$ attitudes in Protestant Developed Countries, with larger $\gamma$ shares in less incomeunequal countries; a prevalence of $\gamma$ attitudes in non-Protestant Christian Developed Countries, with larger $\beta$ shares in more income-unequal countries; a prevalence of $\alpha$ attitudes in Muslim Less Developed Countries, with larger $\gamma$ shares in more educated countries; and a prevalence of $\beta$ attitudes in more educated atheist and Jewish countries.
\end{abstract}

Keywords

Happiness; health; analytical model; attitudes; ethics; culture; religion.

I1, I3, Z1

\section{JEL classification}




\section{Introduction}

Many empirical studies have shown a positive impact of health on happiness. For example, Binder and Coad (2011) associated health status with quintiles for happiness after controlling for income and education. Peiro (2006) compared health status with satisfaction or happiness after controlling for age, marital status, and employment. Pedersen \& Schmidt (2011) examined the effects of the level of and changes in self-reported health on life satisfaction after controlling for the level of and changes in income and labour status. Becchetti \& Rossetti (2009) accounted for the effects of health deterioration on frustrated achievement after controlling for the lack of a full-time job, relative income, marital status shocks, and a poorer social life. Yakovlev \& Leguizamon (2012) examined the effects of high and middle education levels, income, and health on self-reported happiness after controlling for age, trust, stress, temperature, religion, and rainfall.

A few empirical studies have shown a positive impact of happiness on health. For example, Blanchflower \& Oswald (2008) compared blood pressure with happiness across nations. Binder \& Coad (2010) found increases in well-being associated with subsequent increases in health, together with increases in income, marriage, and employment, but decreases in well-being associated with increases in income, marriage, and employment. Diener \& Chan (2011) associated life satisfaction, the absence of negative emotions, optimism, and the presence of positive emotions with better health and longevity.

In addition, many empirical studies have shown significant impacts of various determining factors on both happiness and health. For example, Kiyani et al. (2011) examined the effect of athletic status on mental health and happiness. Oshio \& Kobayashi (2010) examined the effects of regional inequality on happiness and self-rated health after controlling for gender, age, educational attainment, income, occupational status, and political views. Borgonovi (2008) examined the effects of volunteer work on self-reported health and happiness. Andersson (2008) compared selfemployed vs. salaried workers in terms of their job and life satisfaction and mental and general health after controlling for physically and mentally stressful jobs. Gatab \& Pirhayti (2012) examined the effects of regular group exercise on happiness, physical symptoms, depression, social functioning and general health. Salary \& Shaieri (2013) examined the effects of an eccentric personality on psychological dissociation and satisfaction after controlling for social commitment, a positive mood, a feeling of control, and self-consciousness. Haji et al. (2011) examined the effects of life skills training on happiness and psychological health after controlling for emotional regulation, social relationships, and physical situations. Bostani \& Saiiari (2011) examined the effects of an athletic life on stress tolerance and self-assertiveness (combined with happiness) and mental health. Maselko \& Kubzansky (2006) examined the effects of involvement in religious institutions and activities on psychological distress and self-rated health and happiness after controlling for gender. Schatz et al. (2012) examined the effects of receiving a pension during retirement on health and well-being after controlling for gender. Izquierdo (2005) examined the effects of improved acculturation and permanent settlement on a change in health and well-being for the Matsigenka of the Peruvian Amazon. Easterlin (2009) examined the effects of the transition from socialism to capitalism on material living levels, satisfaction with work, health, and family life.

Note that all of these researchers studied the general population and its health; I have not included other studies on the relationship between happiness and health for specific populations such as students in schools (e.g., Shayan \& Gatab, 2012; Yahyaei et al., 2012; Gatab et al., 2011) or patients in hospitals (e.g., Mukuria \& Brazier, 2013). Moreover, these studies applied different definitions and measures of both happiness and health. In the present study, I will not espouse any particular definition of happiness and health nor will I specify any measure of these factors to keep the analysis as general as possible, and I will not account for other studies on the impacts of specific illnesses on happiness (e.g., Binder and Coad, 2013). Finally, all of these researchers studied both happiness and health, so I have disregarded studies that focused only on determinants of one of the two parameters. 
However, my literature review found no analytical studies that described the dynamic interactions between happiness and health. Thus, the first purpose of this paper was to develop a dynamic system of equations that could account for all individual determinants that were investigated by the abovementioned empirical studies, with the goal being to characterise alternative long-run equilibria for happiness and health based on different attitudes to life. I selected very general assumptions; I disregarded all contextual determinants (e.g., temperature or rainfall). A by-product of this first purpose will be a ranking of attitudes in terms of individual health and achievements for a given level of happiness as a function of individual characteristics.

In particular, I will tell the following story:

- Each individual receives a feasible stock $(f s)$ of opportunities from their family, which translates into economic and physical flows of countable opportunities per period. These flows are affected by personal uncertainty $(u)$ and ethical freedom $(f r)$.

- Each individual is aware of the relationship between happiness ( $h a$ ) and health (he). In particular, current health at time $\mathrm{t}(h e[t])$ affects current happiness $(h a[t])$, whereas past happiness $(h a[t-1]$ and $h a[t-2])$ affects current health, with a persistent effect on the personal mood. If people are not aware of this relationship, they could choose paths to happiness and health that instead lead to despair desperation or illness.

- Each individual is aware of the physical and psychological stress $(\delta)$ that arises from improving their economic or social status and from pursuing higher and higher achievements $(y)$; however, this stress might be negligible for some people. Needless to say, $\delta$ refers to perceived inner sensations rather than revealed sentiments, and simulating feelings can actually increase the psychological stress.

- Each individual seeks a long-run equilibrium for happiness and health ( $h a^{*}$ and $\left.h e^{*}\right)$. In particular, if their physical stress is negligible $(\delta=0)$, they will accept oscillations in their achievements, happiness, and health if this leads towards a long-run equilibrium; if their physical stress is positive $(\delta>0)$, they will take actions to move towards their achievement, happiness, and health equilibrium. If people have an incorrect perception of this stress, they may choose paths to achievement, happiness, and health paths that instead lead to despair desperation or illness.

- Each individual can choose from among three alternative attitudes towards life: $\alpha=$ seeking relative improvements in terms of their opportunities, which represents an Aristotelian attitude; $\beta=$ seeking relative increases in achievements per period, which represents an Epicurean attitude; and $\gamma=$ seeking relative improvements in terms of the reference group's average achievements, which represents a Stoic attitude. These choices are made according to the expected values of achievements, happiness, and health or according to the individual's degree of optimism or pessimism for a given educational level, relative economic and social status, ethical freedom, occupation type, and employment status. These approaches could lead to different choices.

Note that this analytical approach is both individually cross-cultural, since it depicts alternative attitudes $(\alpha, \beta, \gamma)$, and socially cross-cultural, since it considers different ethical freedom . Moreover, the model accounts for both ex-ante and ex-post perspectives, as it calculates achievement, happiness, and health at given uncertainty values (i.e., behavioural ex-ante such as "I am optimistic" or behavioural ex-post, such as "an unsolvable problem happened to me"), and the expected achievement, happiness, and calculates health over all values of uncertainty (i.e., statistical ex-ante such as "I am neither optimistic nor pessimistic, and any positive or negative events could happen to me"). Finally, the approach is both individually cross-developmental, as it depicts alternative education and safety occupation levels, and socially cross-developmental; indeed, different income distributions are considered.

The second purpose of this paper was to apply the analytical model to determine how well it can explain the observed differences in health in different countries or cultures in terms of the different ethical freedom and attitudes prevailing in those countries or cultures. Although these characteristics can be debated, they nonetheless represent plausible starting assumptions for this 
analysis. A by-product of this second purpose will be that it provides an empirical test of the analytical model.

\section{The analytical model}

The purpose of this section is to develop mathematical formulas that represent the dynamic interrelationship between happiness $h a[t]$ and health $h e[t]$ at time $t$ based on factors that have been described in the empirical literature. I will refer to the following variables:

- an individual's achievements ( $y$ ) and their reference group's average achievement, ay; one could think of individual income and the income distribution as proxies for $y$ and $a y$, respectively.

- the feasible set $f s$ for opportunities, which in turns depends on the individual flows of income and health that come from the original family ( $f y$ and $f h$, respectively), on personal uncertainty $(u[t])$, and on ethical freedom $(f r)$; one could use family income and health as proxies for $f y$ and $f h$ by solving for $f r$, whereas one could refer to $u[t]$ as negatively or positively affecting the feasible set as a proxy for monetary loss or gain (a change in $f y$ ), injury or recovery (a change in $f h$ ), or any other uncontrolled factors that could reduce or increase the feasible set.

- the employment status $(\mathrm{em})$, the educational level $(\mathrm{ed})$, and the occupation type (oc); one could think of a full-time job, the number of school years, and an unsafe or dangerous job as proxies for $e m, e d$, and $o c$, respectively.

Note that Powdthavee \& van den Berg (2011) highlighted the problems in estimating health in monetary terms using a new well-being valuation method, whereas Lelkes (2006) showed that an individual's self-reported life satisfaction could be used as a proxy for utility, whereas commonly used measures of well-being can bias a person's evaluation of their level of satisfaction. Although the model is sufficiently general to be consistent with many definitions and measures, selfperceived happiness and health seem to be the most appropriate for analyses at an individual level. In this analysis, I will represent the dynamic interrelationship between happiness and health using two dynamic equations, in which normalisations are boldfaced (e.g., fy, fh), and parameters are italicized (e.g., ay, ed, fr, me, oc, em):

$$
\begin{gathered}
h a[t]=\alpha\{(y[t]-f s) / f s\}+\beta\{(y[t]-y[t-1]) / y[t-1]\}+\gamma\{(y[t]-a y) / a y\}+h e[t]+e m \\
h e[t]=f s+\sum_{t-m e} e^{t-1} h a[t]-\delta(y[t]-y[t-1])+y[t]+e d+o c
\end{gathered}
$$

where:

$$
\begin{gathered}
\alpha=1 \text { or } \beta=1 \text { or } \gamma=1 \\
f s=f s[t]-f s[t-1]=\mathbf{f y}+\mathbf{f h}-u[t]+f r \\
\delta \geq 0, o c \leq 0, e m \geq 0, m e \geq 1 \\
u[t] \text { in }\left[-u^{*},+u^{*}\right]
\end{gathered}
$$

where $m e$ is the number of past periods which affect the current the personal mood and $u^{*}$ is the long-run equilibrium uncertainty.

Note that all coefficients are set at 1 . One could introduce alternative values based on specific econometric analyses, although the qualitative insights would not be altered by this choice. Moreover, $f s$ represents the change in the feasible set on which one can rely in the short run; a positive $f s$ implies that $y[t]$ can be 0 or negative. Finally, one could introduce $-u[t]$ or $+e d$ in $h a[t]$, although these are qualitatively irrelevant at equilibrium, but would double the quantitative effects on $h a[t]$, since these variables linearly affect $f s$ and $h e[\mathrm{t}]$, respectively

Equations (1) and (2) provide a theoretical framework that justifies the many relationships observed between the many variables involved in a linear way. The goals are to obtain an analytical solution, permit the use of graphs to reveal insights, achieve an immediate empirical validation, and enable future econometric analyses. Alternatively, one could think of equations (1) and (2) as a linearized and discretised globally stable solution for the following inter-temporal optimisation problem: 


\section{Choose $y[t]$ and then $\alpha=1$ or $\beta=1$ or $\gamma=1$ to maximise \\ $\int_{0}^{\infty} h a(y[t], y[t-1], u[t], f r, h e[t], e m) e^{\varepsilon t} \mathrm{dt}$ \\ s.t. $(\partial h e / \partial t)\{h a[t-1], y[t], y[t-1], e d, o c\}=0$}

where $\varepsilon=0$ implies a co-state variable fixed at $\infty$ and $\varepsilon=\infty$ implies a co-state variable fixed at 0 , whenever the impact of he on $h a$ is linear. However, this would require the implausible assumptions of complete information and inter-temporal rationality.

Note that many of the parameters and coefficients that I have applied have been estimated in the literature. For example, Golden \& Wiens-Tuers (2006) showed that mandatory and harmful overtime work $(\delta>0)$ has a negative impact on self-reported subjective happiness and psychological health after controlling for income. Verme (2009) shows that freedom of choice predicts life satisfaction better than any other known factor, including health, employment, income, marriage, or religion, both across and within countries. Graham et al. (2004) showed that self-esteem and optimism improved both wealth and health after controlling for income level, health status, and some other factors. Carlsson et al. (2014) showed an intergenerational transmission of happiness after controlling for the income, gender, education, health status, marital status, and well-being of the parents. Gardner \& Oswald (2007) estimated a positive impact of income on psychological health. Van Campen \& Cardol (2009) showed that physical disabilities reduce happiness through participation in paid and volunteer work after controlling for education and age. Brown \& Tierney (2009) showed a strong negative relationship between religious participation and subjective wellbeing after controlling for demographics, health and disabilities, living arrangements, wealth, and income. Guney (2011) showed that individuals could be helped to discover strengths such as optimism, hope, humour, and resilience.

In addition, achievements are more likely to be closer to the $f$ s border for $\beta$ and $\gamma$ types of attitude than for the $\alpha$ type. By stressing that ethical freedom and its social implications (i.e., $f r$ ) are distinguished from personal attitudes that lack social implications (i.e., $\alpha, \beta$, and $\gamma$ attitudes), in this analysis, I will refer to Zagonari (2009) that predicted a negative $f r$ for members of Protestant religions and a positive $f r$ for members of non-Protestant Christian, Jewish, Muslim, Buddhist, and Hindu religions and for atheists; in addition, I have associated the $\alpha, \beta$, and $\gamma$ attitudes with the Aristotelian, Epicurean, and Stoic attitudes to life, respectively. Indeed, $\alpha$ evokes the flourishing of personal potentials (i.e., a percentage increase of one's feasible set), $\beta$ is attached to an ongoing percentage increase in achievements (i.e., meeting one's current goals or desires), and $\gamma$ evokes the awareness of a common destiny (i.e., a percentage increase over the average conditions of other individuals within one's group).

\section{Analytical results}

The previous section provided mathematical formulas that represent the dynamic interrelationship between happiness $(h a[t])$ and health $(h e[t])$ at time $t$. In this section, I will characterise the equilibrium values for happiness $\left(h a^{*}\right)$ and health $\left(h e^{*}\right)$. I will assume that $h a[t]=h a^{*}$ for each $t$ and, consequently, that $\sum_{t-m e}{ }^{t-1} h a[t]=m e \times h a^{*}$. Note that the case in which $\delta>0$ and $y[t]-y[t-1] \neq 0$ can be rejected as implausible; if an individual suffers from a change in achievements $(\delta>0)$, they will seek to avoid changes, at least at each time $t$. Next, $y$ is not constrained, since one's income can increase to any extent, and one could also sell their economic or health flows.

\subsection{The equilibrium if $\delta=0, y[t]-y[t-1] \neq 0$, and $\beta=1$}

Let us solve equation (2) for $y[t]$ and for $y[t-1]$ in terms of $h e^{*}$ :

$$
\begin{gathered}
y[t]=-e d-f h-f y+h e^{*}-m e \text { ha-fr-oc+u[t]} \\
y[t-1]=-e d-f h-f y+h e^{*}-m e \text { ha-fr-oc+u[t-1]}
\end{gathered}
$$

Let us use these equations in equation (1) for a given $h a$ to obtain the equilibrium $u^{*}$ : 


$$
u^{*}=u[t]=u[t-1] \text { iff } u^{*}=e d+e m+f h+f y-h e^{*}+m e h a+o c+f r
$$

However, $y[t]=y[t-1]=y^{*}=0$ at $u=u^{*}$. Thus, if $\beta=1, h a^{*}=h e^{*}+e m$ or $h e^{*}=h a^{*}-m e$, and the equilibrium $u^{*}$ becomes:

$$
u^{*}=u[t]=u[t-1]=e d+e m+f h+f y-h a^{*}+m e h a^{*}+o c+f r
$$

whereas $u[t]$ as a function of $u[t-1]$ becomes:

$$
u[t]=\left\{-\left[e d o c+e m o c+f h o c+f y o c+o c^{2}+(-o c+m e o c) h a^{*}+o c f r\right] /(1-o c)\right\}+\{u[t-1] /(1-o c)\}
$$

Let us check for the stability conditions of $u[t]$ in terms of $u[t-1]$ :

$$
\begin{gathered}
\mathrm{d} u[t] / \mathrm{d} u[t-1]=1 /(1-o c) \\
u[0]=-\left[e d o c+e m o c+f h o c+f y o c+o c^{2}+h a^{*}(-o c+m e o c)+o c f r\right] /(1-o c)
\end{gathered}
$$

Thus, $u^{*}$ is globally stable if $u[t]$ is increasing but at a relatively low rate $(o c<-1)$, either with a positive y-axis intercept (oc>-ed-em-fh-fy+ha-ha me-fr), a negative y-axis intercept (oc<-ed-em$f h-f y+h a-h a m e-f r)$, or if $u[t]$ is decreasing but at a relatively low rate $(o c>1)$, with a positive y-axis intercept (oc>-ed-em-fh-fy+ha-ha me-fr). Note that $h e^{*}$ does not depend on $o c$ at $u=u^{*}$.

In other words, if one chooses to have a $\beta$ attitude, one accepts both changing happiness and health levels by increasing achievements in the case of unlucky events, and vice versa. However, it is implausible to accept infinitely divergent changes in both happiness and health. Thus, this individual will look for a convergent path, and will choose to be a $\beta$ attitude (a) if they adopt a behavioural ex-ante approach, where $u^{*}>0$ requires them to be optimistic and $u^{*}<0$ requires them to be pessimistic; (b) if they adopt a statistical ex-ante approach, where $u^{*}=0$; or (c) if they adopt a behavioural ex-post approach, where $u^{*}>0$ requires them to be unlucky and $u^{*}<0$ requires them to be lucky. Note that a change in psychological status (from optimistic to pessimistic or vice versa) or in contextual events (from lucky to unlucky or vice versa) might require a change in the attitude type to avoid long-run disequilibria.

\subsection{The equilibrium if $\delta>0, y[t]-y[t-1]=0$, and $\alpha=1$ or $\mathrm{y}=1$}

By substituting equation (2) into equation (1) and solving for $y^{*}$, we obtain:

$$
y *=\frac{a y(f h+f y+f r-u)(\alpha-e m-e d-f h-f y+\gamma+h a-h a \times m e-f r+u)}{f y \times \gamma+\alpha \times a y+f y \times a y+f h(\gamma+a y)+\gamma \times f r+a y \times f r-(\gamma+a y) u}
$$

We can then substitute $y^{*}$ into equation (2) to obtain $h e^{*} \geq 0$ :

$$
\begin{aligned}
& h e * \\
& =e d+f h+f y+h a \times m e+o c+f r-u \\
& -\frac{a y(f h+f y+f r-u)(-\alpha+e m+e d+f h+f y-\gamma-h a+h a \times m e+f r-u)}{f y \times \gamma+\alpha \times a y+f y \times a y+f h(\gamma+a y)+\gamma \times f r+a y \times f r-(\gamma+a y) u}
\end{aligned}
$$

where $h a^{*}=h a$. Note that $u$ is assumed to range from $-u^{*}$ to $+u^{*}$, whereas $h e^{*}=e d+f h+f y+h a$ $m e+o c+f r-u+y^{*}$ is independent of $a y$, and $y^{*}$ is independent of $o c$.

In other words, if one chooses $\alpha$ or $\gamma$ attitudes, one rejects changing happiness and health levels by fixing equilibrium achievements according to one's psychological status or contextual events. In particular, this individual will choose an $\alpha$ or $\gamma$ attitude (a) if they adopt a behavioural ex-ante approach, they will choose $y^{*}, h e^{*}$, and $h a^{*}$ with $u>0$ if they are optimistic, or with $u<0$ if they are pessimistic; (b) if they adopt a statistical ex-ante approach, they will choose $y^{*}, h e^{*}$, and ha* with 
$u=0$; and (c) if they adopt a behavioural ex-post approach, they will choose $y^{*}, h e^{*}$, and $h a^{*}$ with $u>0$ if they are unlucky, versus $u<0$ if they are lucky. Note that a change in psychological status (from optimistic to pessimistic or vice versa) or in contextual events (from lucky to unlucky or vice versa) might require a change in the equilibrium achievements rather than a change in the attitude type, although a change in achievements will affect their health in the short-run.

\section{Reasoned insights}

The previous section characterised the equilibrium values for achievements $\left(y^{*}\right)$, happiness $\left(h a^{*}\right)$, and health $\left(h e^{*}\right)$ for the three attitudes $(\alpha, \beta$, and $\gamma)$. In this section, I will apply these equilibrium values to obtain rankings of the three attitudes in terms of $y^{*}, h a^{*}$, and $h e^{*}$ and, consequently, will provide insights into which attitude should be adopted by each individual, according to their characteristics, and by citizens of each country, according to its characteristics.

\subsection{At an individual level}

In this section, I will present analytical results and graphical insights at an individual level. Let us normalise, without loss of generality, $f y=h a^{*}=1$ (i.e., define a unitary measure), as well as $f h=1$ and $e m=0$ (i.e., the $h e^{*}$ axis is fixed at 1). Table 1 presents the first, second, and cross derivatives of the optimal $y^{*}$ and $h e^{*}$ with respect to $f r, e d$, and $a y$, with the other parameters and variables fixed at a reference case to make all cases comparable. An increase in ethical freedom $(\partial f r>0)$ and an increase in education $(\partial e d>0)$ would result in the same level of happiness and health, with smaller achievements $\left(\partial y^{*}<0\right)$, regardless of attitudes, whereas an increase in the achievements of others $(\partial a y>0)$ would require an increase in individual achievements $\left(\partial y^{*}>0\right)$ for a $\gamma$ attitude only, unless the uncertainty is sufficiently small, in which case people may reach the same status through high luck rather than high achievement. In contrast, an increase in ethical freedom $(\partial f r>0)$ and an increase in education $(\partial e d>0)$ would result in the same level of happiness and a higher level of health $\left(\partial h e^{*}>0\right)$, with the same achievements, regardless of the attitude, whereas an increase in the achievements of others $(\partial a y>0)$ would require an increase in the individual health $\left(\partial h e^{*}>0\right)$ for a $\gamma$ attitude only, unless the uncertainty is sufficiently small. Moreover, the second and cross derivatives are the same and are symmetrical for $y^{*}$ and $h e^{*}$; this means that indirect effects on $h e^{*}$ arise from effects on $y^{*}$. Finally, an increase in ethical freedom $(\partial f r>0)$ and an increase in education $(\partial e d>0)$ would result in the same level of happiness and health with smaller achievements $\left(\partial y^{*}<0\right)$ at an increasing rate (i.e., both decreasing and convex functions) for an $\alpha$ attitude, whereas for a $\gamma$ attitude, an increase in the achievements of others ( $\partial a y>0)$ would require an increase in individual achievements $\left(\partial y^{*}>0\right)$ at an increasing rate (i.e., an increasing and convex function), unless the uncertainty is sufficiently small, but at a decreasing rate if combined with a decrease in ethical freedom $(\partial f r>0)$ and an increase in education $(\partial e d>0)$ (i.e., both increasing and concave functions).

Table 1.First, second, and cross derivatives of $y^{*}$ and $h e^{*}$ at the reference case values $(f r=0, e d=0, a y=1, f y=1$, $f h=1, m e=1, e m=0)$ for alternative attitudes $(\alpha=1, \beta=1, \gamma=1)$ with $u$ in $\left[-u^{*},+u^{*}\right]$.

\begin{tabular}{|c|c|c|c|c|c|}
\hline & $\partial y^{*}$ & $\partial h e^{*}$ & $\partial y^{*} / \partial f r$ & $\partial y^{*} / \partial e d$ & $\partial y^{*} / \partial a y$ \\
\hline & & & & & \\
\hline$\partial f r$ & $\left(-7+6 u-u^{2}\right) /(3-u)^{2},-1,-1 / 2$ & $2 /(3-u)^{2}, 0,1 / 2$ & $-4 /(3-u)^{3}, 0,0$ & $-1 /(3-u)^{2}, 0,0$ & $0,0,-1 / 4$ \\
\hline$\partial e d$ & $-[(2-u) /(3-u)],-1,-1 / 2$ & $1 /(3-u), 0,1 / 2$ & $-1 /(3-u)^{2}, 0,0$ & $0,0,0$ & $0,0,-1 / 4$ \\
\hline$\partial a y$ & $0,0,1 / 4(-1+u)$ & $0,0,1 / 4(-1+u)$ & $0,0,-1 / 4$ & $0,0,-1 / 4$ & $0,0,1 / 4(1-u)$ \\
\hline
\end{tabular}

Let us apply a standard normal distribution to $u$, with $\mu=0$ and $\sigma=1$. Figure 1 to Figure 18 present the results of numerical simulations with large ranges of parameter values compared to the values in the reference case, to depict specific conditions at an individual level, with the parameters normalised to make all cases comparable. Insights based on the average values can be rejected as implausible, since they would require risk-neutral individuals who are willing to bet on their lives. Note that if $o c=0$, any $u$ for $\beta=1$ will represent an equilibrium. Moreover, fixing $h a^{*}$ at 1 relies on the over-determinacy of the dynamic model, with two equilibrium equations and three control 
variables. Finally, $m e$ is set to 1 , since larger values together with $h a^{*}=1$ amount to larger education levels.

Figure 1. The reference case. Blue if $\alpha=1$, point if $\beta=1$, purple if $\gamma=1 ;$ ay=1 (average income); $e d=0$ (middle education); $f r=0$ (average ethical freedom); oc (safe occupation) $=0$.

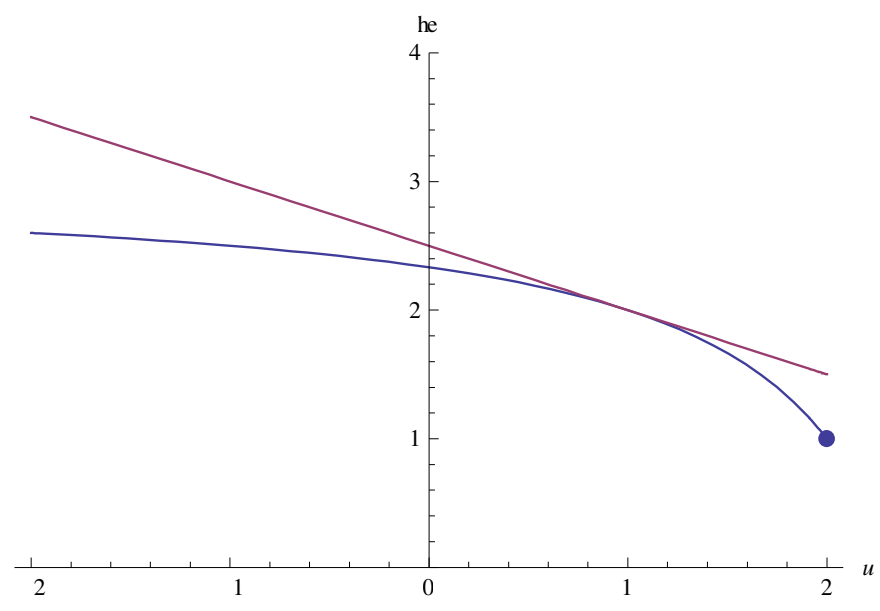

Figure 2. The reference case. Blue if $\alpha=1$, point if $\beta=1$, purple if $\gamma=1$; ay=1 (average income); $e d=0$ (middle education); $f r=0$ (average ethical freedom); $o c$ (safe occupation) $=0$.

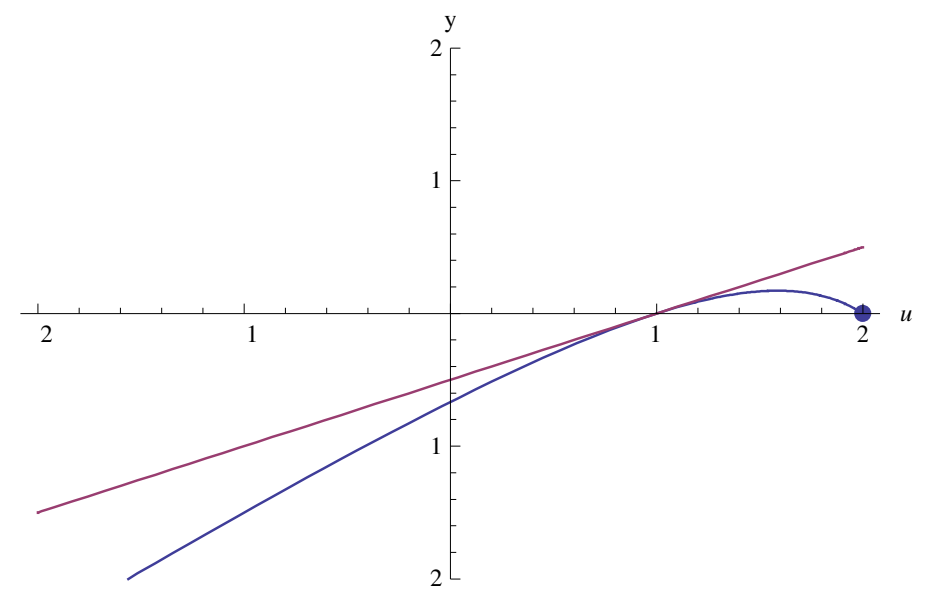

Figure 3. The reference case. Blue if $\alpha=1$, point if $\beta=1$, purple if $\gamma=1$; ay=1 (average income); $e d=0$ (middle education); $f r=0$ (average ethical freedom); $o c$ (unsafe occupation) $=-1.5$.

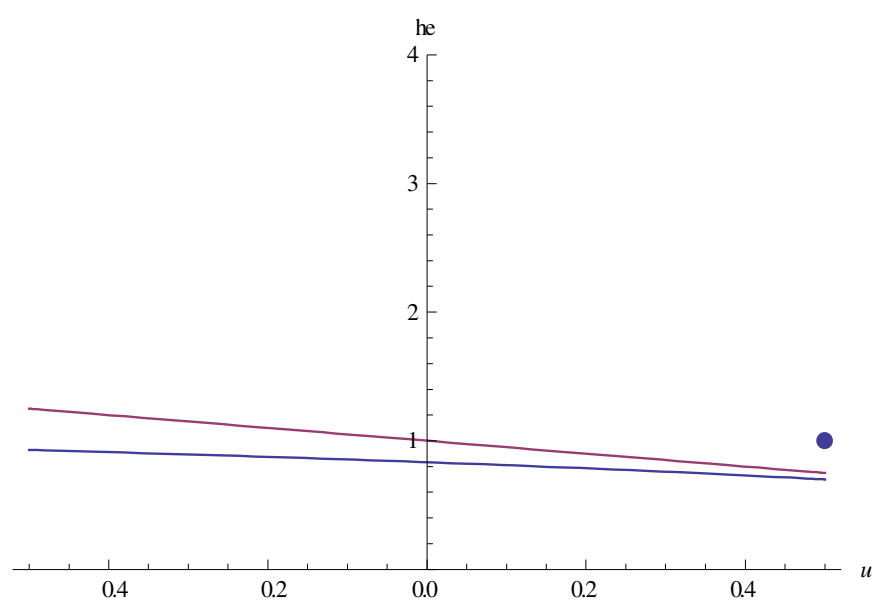


Figure 4. A non-Protestant society. Blue if $\alpha=1$, point if $\beta=1$, purple if $\gamma=1$; $a y=1$ (average income); $e d=0$ (middle education); $f r=1.5$ (high ethical freedom); $o c=0$ (safe occupation).

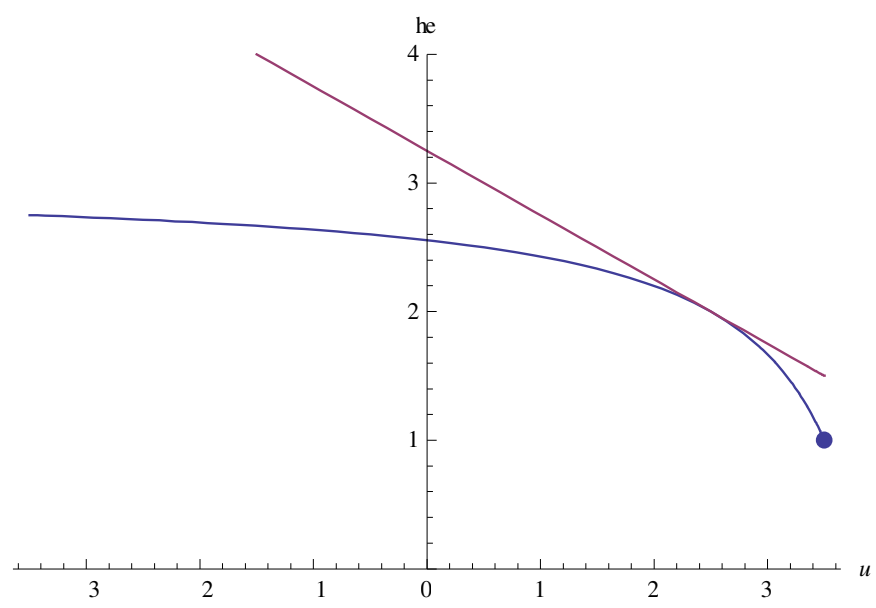

Figure 5. A non-Protestant society. Blue if $\alpha=1$, point if $\beta=1$, purple if $\gamma=1$; $a y=1$ (average income); $e d=0$ (middle education); $f r=1.5$ (high ethical freedom); $o c=0$ (safe occupation).

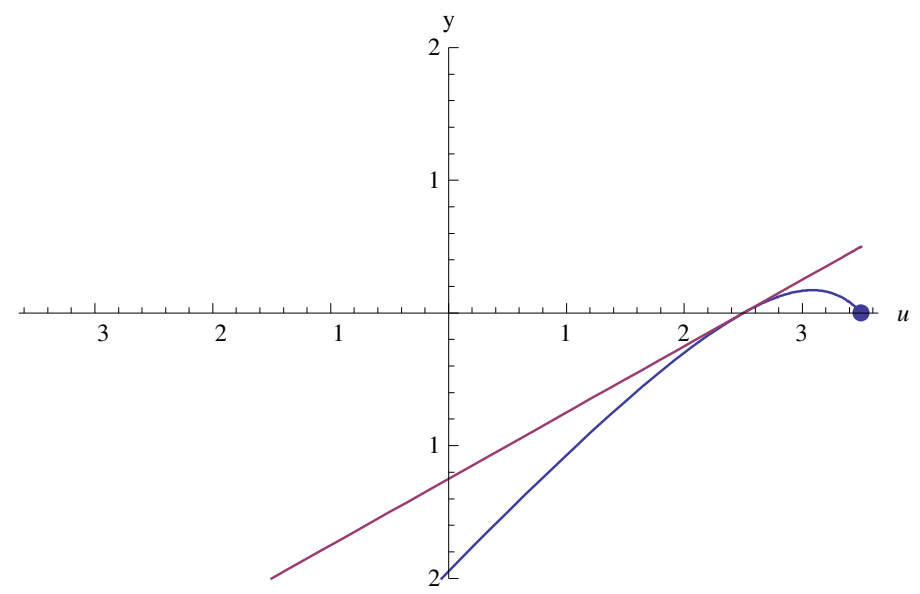

Figure 6. A non-Protestant society. Blue if $\alpha=1$, point if $\beta=1$, purple if $\gamma=1$; $a y=1$ (average income); $e d=0$ (middle education); $f r=1.5$ (large ethical freedom); $o c=-1.5$ (unsafe occupation).

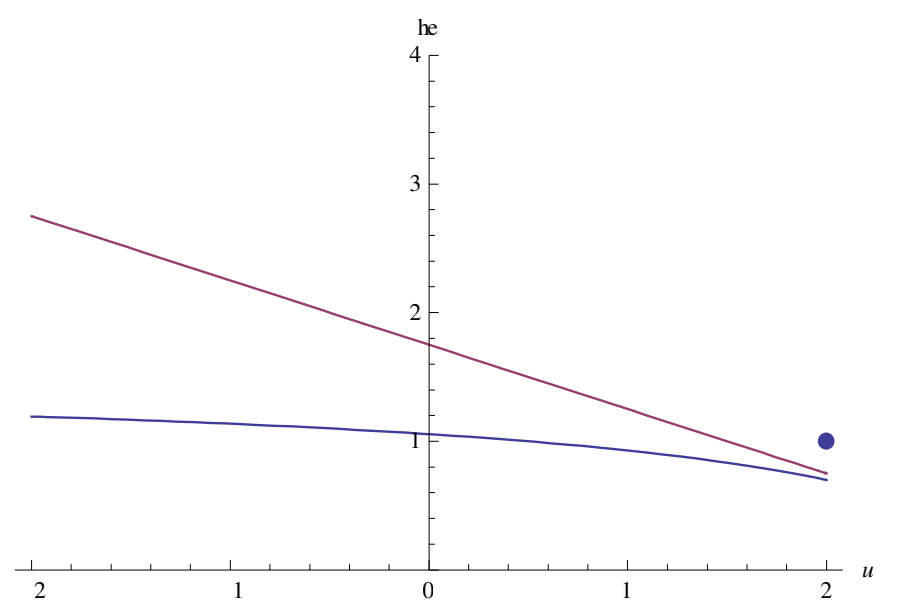


Figure 7. A Protestant society. Blue if $\alpha=1$, point if $\beta=1$, purple if $\gamma=1$; $a y=1$ (average income); $e d=0$ (middle education); $f r=-1.5$ (low ethical freedom); $o c=0$ (safe occupation).

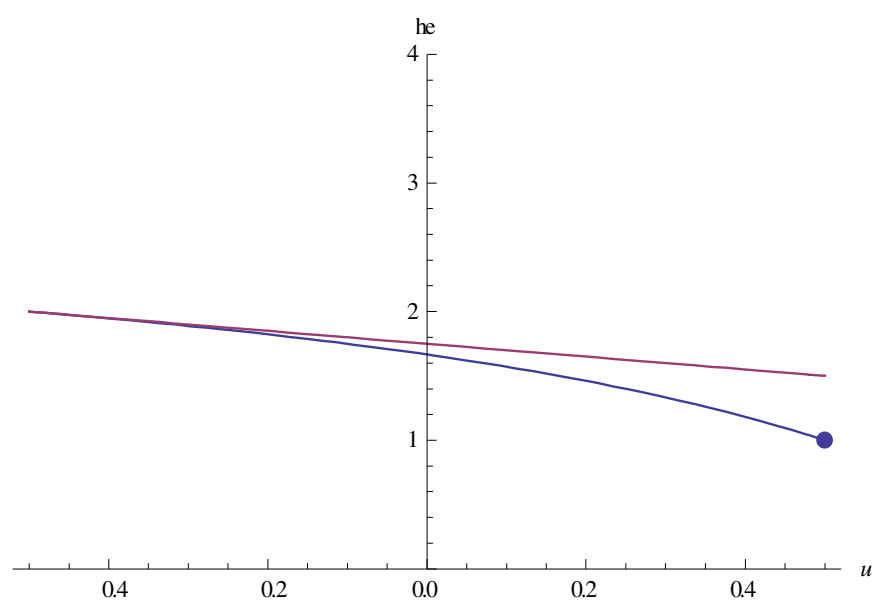

Figure 8. A Protestant society. Blue if $\alpha=1$, point if $\beta=1$, purple if $\gamma=1$; $a y=1$ (average income); $e d=0$ (middle education); $f r=-1.5$ (low ethical freedom); $o c=0$ (safe occupation).

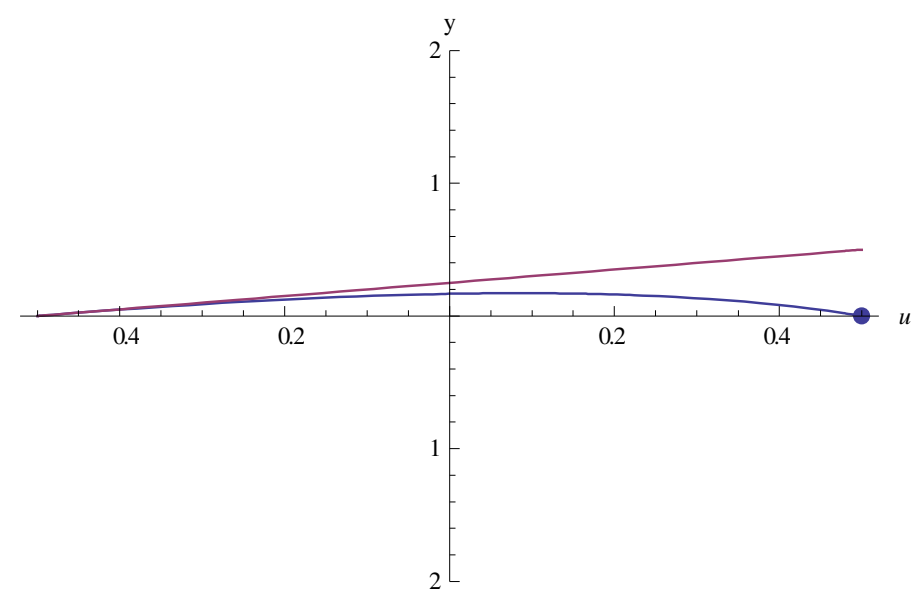

Figure 9. A Protestant society. Blue if $\alpha=1$, point if $\beta=1$, purple if $\gamma=1$; $a y=1$ (average income); $e d=0$ (middle education); $f r=-1.5$ (low ethical freedom); $o c=-1.5$ (unsafe occupation).

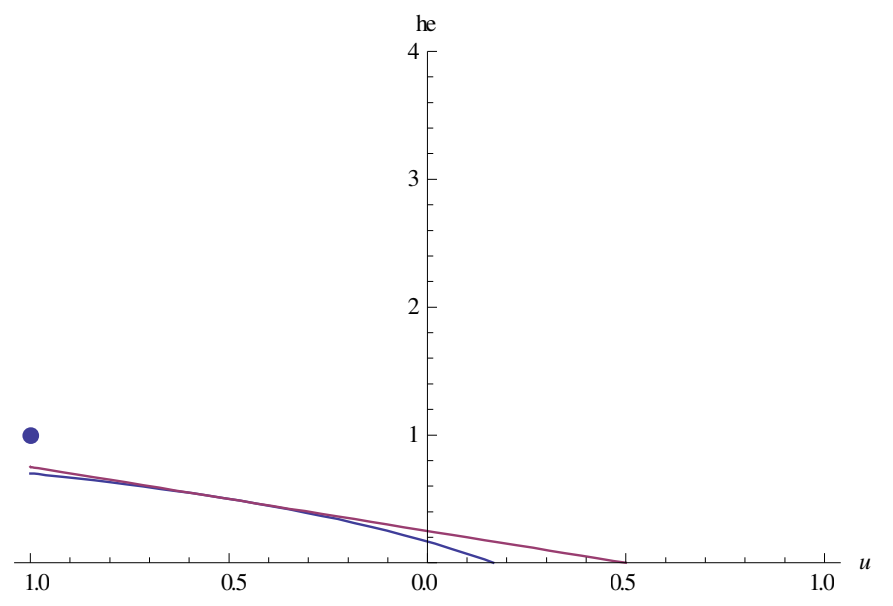


Figure 10. Low education. Blue if $\alpha=1$, point if $\beta=1$, purple if $\gamma=1$; $a y=1$ (average income); $e d=-2$ (low education); $f r=0$ (average ethical freedom); $o c=0$ (safe occupation).

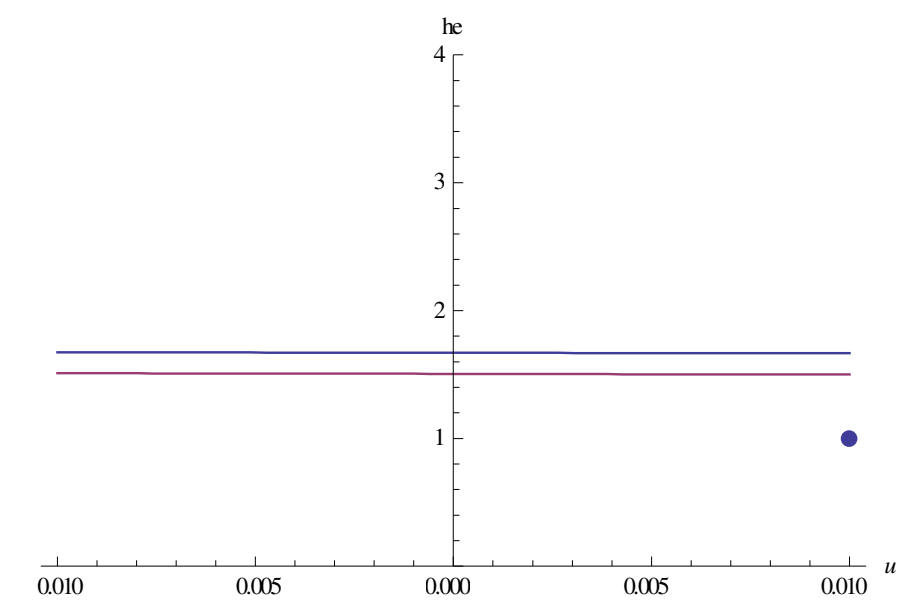

Figure 11. Low education. Blue if $\alpha=1$, point if $\beta=1$, purple if $\gamma=1$; ay=1 (average income); $e d=-2$ (low education); $f r=0$ (average ethical freedom); $o c=0$ (safe occupation).

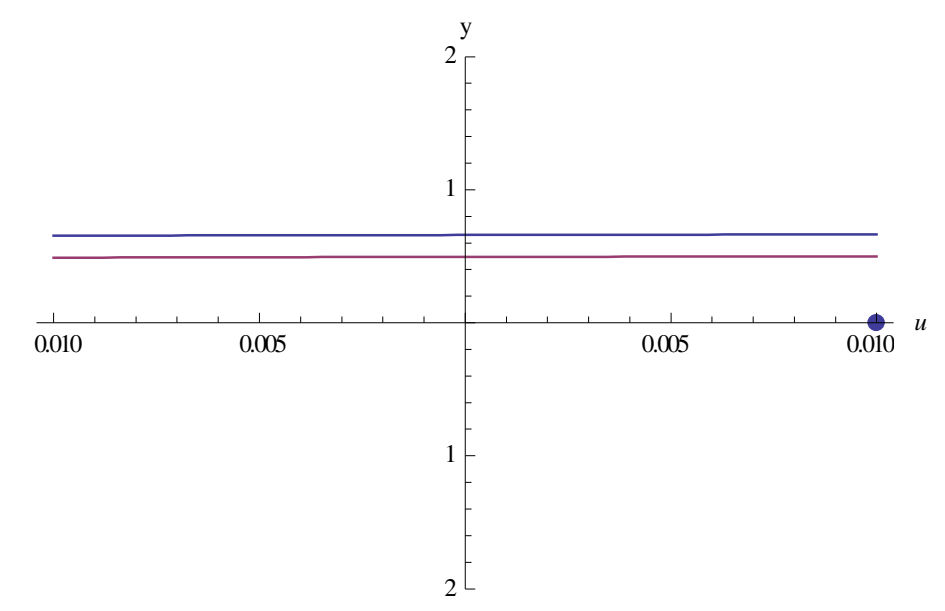

Figure 12. Low education. Blue if $\alpha=1$, point if $\beta=1$, purple if $\gamma=1$; ay=1 (average income); $e d=-2$ (low education); $f r=0$ (average ethical freedom); oc =-1.5 (unsafe occupation).

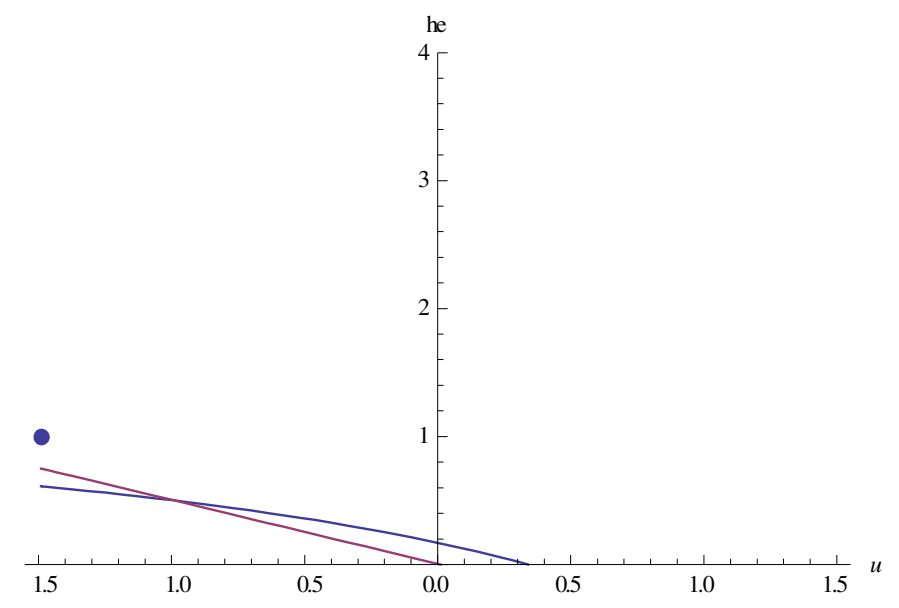


Figure 13. High education. Blue if $\alpha=1$, point if $\beta=1$, purple if $\gamma=1$; $a y=1$ (average income); $e d=2$ (high education); $f r=0$ (average ethical freedom); $o c=0$ (safe occupation).

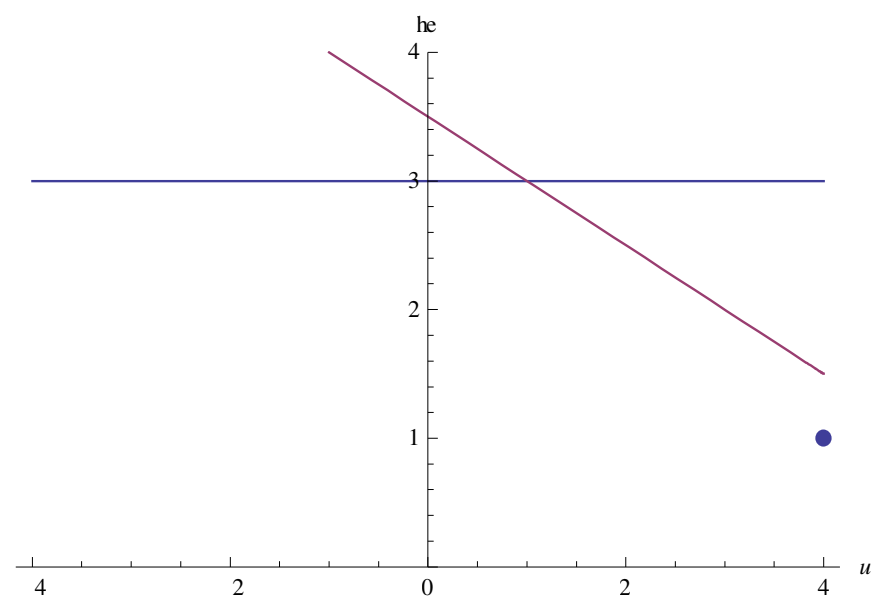

Figure 14. High education. Blue if $\alpha=1$, point if $\beta=1$, purple if $\gamma=1$; $a y=1$ (average income); $e d=2$ (high education); $f r=0$ (average ethical freedom); $o c=0$ (safe occupation).

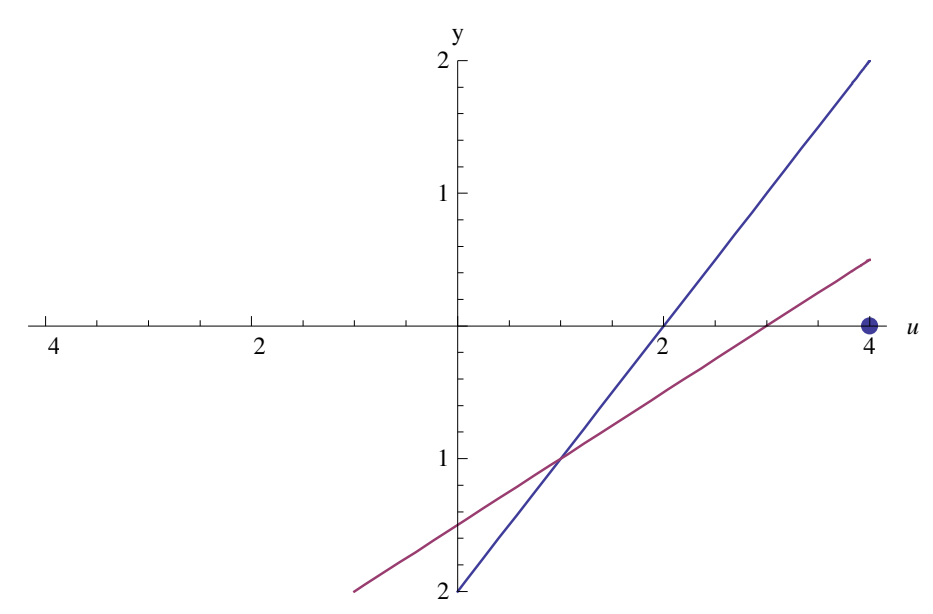

Figure 15. High education. Blue if $\alpha=1$, point if $\beta=1$, purple if $\gamma=1$; $a y=1$ (average income); $e d=2$ (high education); $f r=0$ (average ethical freedom); oc =-1.5 (unsafe occupation).

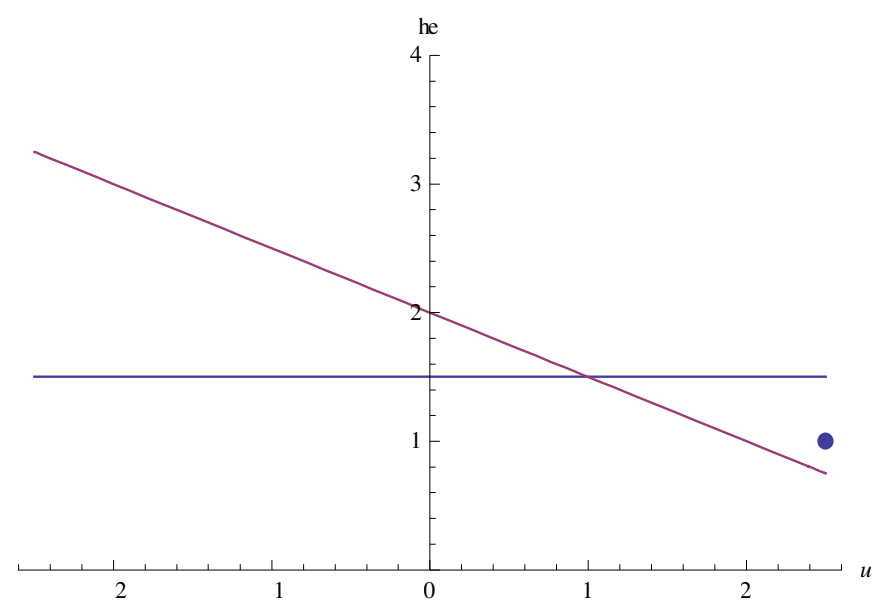


Figure 16. An income-unequal society. Blue if $\alpha=1$, point if $\beta=1$, purple if $\gamma=1 ; a y=10$ (small income); $e d=0$ (middle education); $f r=0$ (average ethical freedom); $o c=0$ (safe occupation).

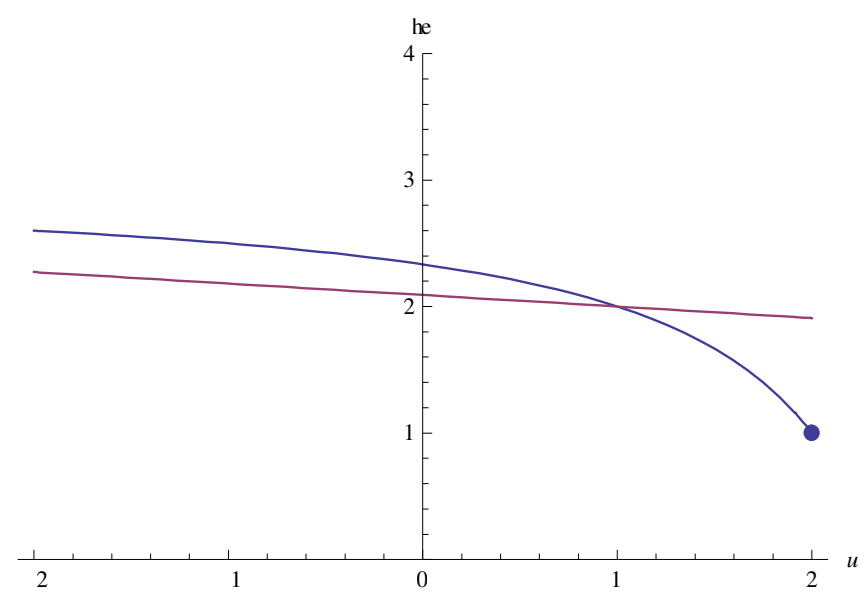

Figure 17. An income-unequal society. Blue if $\alpha=1$, point if $\beta=1$, purple if $\gamma=1 ; a y=10$ (small income); $e d=0$ (middle education); $f r=0$ (average ethical freedom); $o c=0$ (safe occupation).

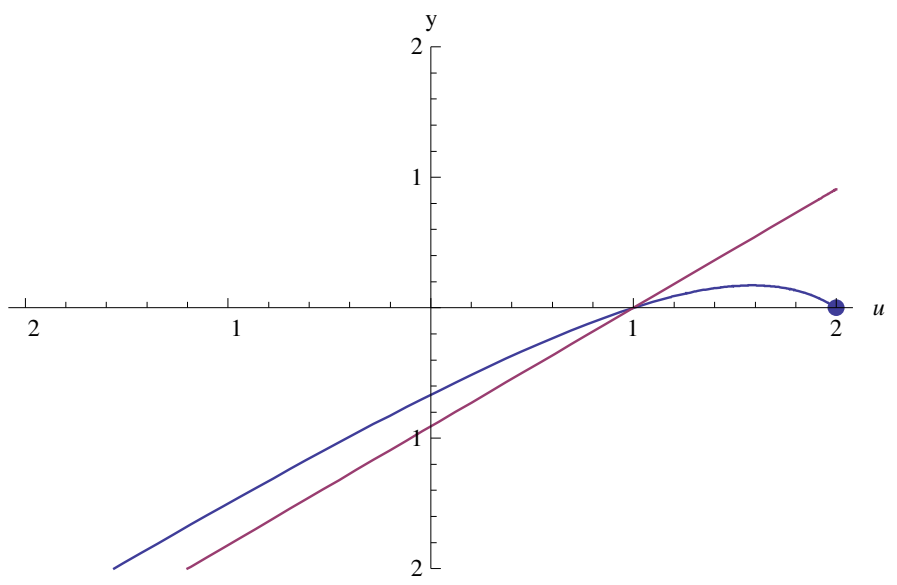

Figure 18. An income-unequal society. Blue if $\alpha=1$, point if $\beta=1$, purple if $\gamma=1 ; a y=10$ (small income); $e d=0$ (middle education); $f r=0$ (average ethical freedom); $o c=-1.5$ (unsafe occupation).

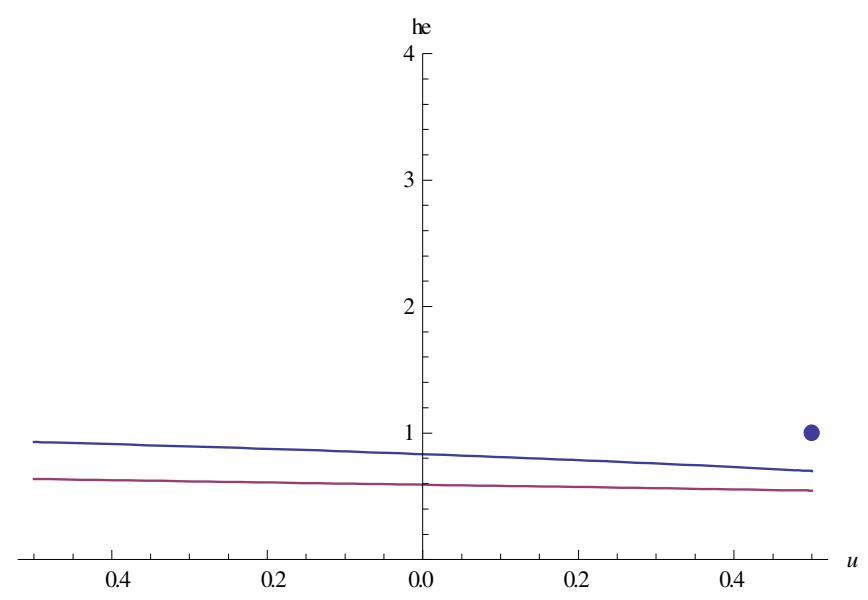

The behavioural ex-ante approach can be described with examples: an individual with a low educational level ( $e d=-2)$ should follow an $\alpha$ attitude if they are pessimistic $(u>1)$, but a $\gamma$ attitude if they are optimistic $(u<1)$.

The statistical ex-ante approach, based on expected values, leads to the following insights: 
- In the reference case, for health (Figure 1), $2.38 \gamma>2.15 \alpha>1 \beta$, whereas for achievements (Figure 2), $2 \beta>1.53 \gamma>1.3 \alpha$. However, if the occupation is unsafe (Figure 3), $1 \beta>0.38 \gamma>0.31 \alpha$ and 2 $\beta>1.81 \gamma>1.75 \alpha$ for health and achievements, respectively.

- In a non-Protestant society, for health (Figure 4), $3.24 \gamma>2.52 \alpha>1 \beta$, whereas for achievements (Figure 5), $2 \beta>0.76 \gamma>0.04 \alpha$. However, if the occupation is unsafe (Figure 6), $1.67 \gamma>1 \beta \approx 0.98$ $\alpha$ and $2 \beta>0.81 \gamma>0.13 \alpha$ for health and achievements, respectively.

- In a Protestant society, for health (Figure 7), $1 \beta>0.67 \gamma>0.61 \alpha$, whereas for achievements (Figure 8), $2.09 \gamma \approx 2.04 \alpha \approx 2 \beta$. However, if the occupation is unsafe (Figure 9), $1 \beta>0.04 \alpha>-0.17$ $\gamma$ and $2.04 \alpha \approx 2 \beta>1.83 \gamma$ for health and achievements, respectively.

- For individuals with a low level of education, for health (Figure 10), $1.36 \gamma \approx 1.34 \alpha>1 \beta$, whereas for achievements (Figure 11), $2 \beta=2 \gamma \approx 1.98 \alpha$. However, if the occupation is unsafe (Figure 12), $1 \beta>-0.18 \alpha>-0.19 \gamma$ and $2 \alpha=2 \beta=2 \gamma$ for health and achievements, respectively.

- For individuals with a high level of education, for health (Figure 13), $3.49 \gamma>2.99 \alpha>1 \beta$, whereas for achievements (Figure 14), $2 \beta>0.51 \gamma>0.01 \alpha$. However, if the occupation is unsafe (Figure 15), $1.97 \gamma>1.48 \alpha>1 \beta$ and $2 \beta>0.52 \gamma>0.03 \alpha$ for health and achievements, respectively.

- In poor families, for health (Figure 16), $2.15 \alpha>1.99 \gamma>1 \beta$, whereas for achievements (Figure 17), $2 \beta>1.30 \alpha>1.14 \gamma$. However, if the occupation is unsafe (Figure 18), $1 \beta>0.31 \alpha>0.22 \gamma$ and $2 \beta>1.75 \alpha>1.66 \gamma$ for health and achievements, respectively.

The behavioural ex-post approach can be described with examples: a well-educated individual $(e d=2)$ who has problems without solutions $(u>0)$ should adopt an $\alpha$ attitude.

Therefore, ceteris paribus, the model suggests that individuals in Protestant and non-Protestant societies should adopt a $\beta$ attitude and a $\gamma$ attitude, respectively (i.e., $\partial \partial h e^{*} / \partial f r \partial \alpha>0$ and $\partial \partial h e^{*} / \partial f r \partial \gamma>0$ with $\partial \partial h e^{*} / \partial f r \partial \alpha<\partial \partial h e^{*} / \partial f r \partial \gamma$ ); educated individuals should adopt a $\gamma$ attitude (i.e., $\partial \partial h e^{*} / \partial e d \partial \alpha>0$ and $\partial \partial h e^{*} / \partial e d \partial \gamma>0$ with $\left.\partial \partial h e^{*} / \partial e d \partial \alpha<\partial \partial h e^{*} / \partial e d \partial \gamma\right)$; and poor individuals should adopt an $\alpha$ attitude (i.e., $\partial \partial h e^{*} / \partial a y \partial \alpha>0$ and $\partial \partial h e^{*} / \partial$ ay $\partial \gamma>0$ with $\partial \partial h e^{*} / \partial a y \partial \alpha>\partial \partial h e^{*} / \partial a y \partial \gamma$ ).

Note that if a $\beta$ attitude begins with $y[t]$ larger than the equilibrium value, then in the short-run, they could obtain larger achievements in their pathways towards equilibrium. Moreover, a persistent unlucky event could compromise an individual's health status, regardless of their chosen attitude, particularly if combined with an unsafe occupation. Finally, the convergent path towards equilibrium for a $\beta$ attitude depends on their life experiences (i.e., a long-run equilibrium might not exist if optimistic individuals face unlucky events or vice versa and then someone with a $\beta$ attitude could move to $\alpha$ or $\gamma$ attitudes), whereas any life experience is consistent with reaching an equilibrium for $\alpha$ and $\gamma$ attitudes.

\subsection{At a country level}

Table 2 summarises the suggested attitudes with small ranges of parameter values compared to the values in the reference case, to depict representative individuals at a country level.

Table 2.Suggested attitudes (bold) based on two sets of social characteristics (education and equity).

\begin{tabular}{|c|c|c|c|c|c|}
\hline & & \multicolumn{2}{|c|}{ DCs $(o c=0)$} & \multicolumn{2}{|c|}{ LDCs $(o c=-1)$} \\
\hline & Education & $a y=1$ (equal) & $a y=10$ (unequal) & $a y=1$ (equal) & $a y=10$ (unequal) \\
\hline \multirow{3}{*}{$\begin{array}{c}\text { Protestant } \\
(f r=-1.5)\end{array}$} & $\operatorname{High}(e d=1)$ & $1.94 \gamma>1 \boldsymbol{\beta}>0 \alpha$ & & & \\
\hline & Middle $(e d=0)$ & & $1 \boldsymbol{\beta}>0.74 \gamma>0.61 \alpha$ & & \\
\hline & Low $(e d=-1)$ & & & & \\
\hline$f r=0$ & High $(e d=1)$ & & & & \\
\hline$f r=0$ & Middle $(e d=0)$ & & & & \\
\hline$f r=0$ & Low $(e d=-1)$ & & $1.08 \boldsymbol{\alpha} \approx 1.06 \gamma \approx 1 \boldsymbol{\beta}$ & & $1 \boldsymbol{\beta}>0 \alpha>0 \gamma$ \\
\hline \multirow{3}{*}{$\begin{array}{l}\text { Non-Protestant } \\
\quad(f r=1.5)\end{array}$} & High $(e d=1)$ & $3.74 \gamma>1 \beta>0 \alpha$ & & & $1.76 \alpha>1.31 \gamma>1 \beta$ \\
\hline & Middle $(e d=0)$ & & & & \\
\hline & Low $(e d=-1)$ & & & & $1.43 \alpha>1 \beta \approx 0.98 \gamma$ \\
\hline
\end{tabular}

Therefore, under the assumption that individuals are observed at their long-run equilibrium attitude, the model predicts a prevalence of $\beta$ and $\gamma$ attitudes in highly educated Protestant DCs, with a larger 
proportion of $\beta$ in more income-unequal countries; a prevalence of $\gamma$ attitudes in non-Protestant Christian DCs; a prevalence of $\alpha$ attitudes in income-unequal LDCs with low educational levels; and a prevalence of $\alpha$ and $\gamma$ attitudes in income-unequal LDCs with high educational levels.

\section{An empirical test of the model}

The previous section provided insights into which attitudes should be adopted by each individual according to their characteristics, and which attitudes should be adopted by each society according to its characteristics. In this section, I will compare these predictions with observed achievements and health to provide an empirical test of the analytical model.

In this analysis, I will consider equations (1) and (2) from section 2 as depicting a representative individual at a country level, where $\alpha, \beta$, and $\gamma$ measure the proportion of a country's population who adopt each attitude type (i.e., $\alpha+\beta+\gamma=1$ instead of $\alpha=1$ or $\beta=1$ or $\gamma=1$ ). Moreover, I will assume that $u=0$ and evaluate $y^{*}$ and $h e^{*}$ at $u=0$ by using an average value approach for the parameter $u$. Finally, I will use life expectancy at birth (LEB) as an indicator of health, average values of per capita GDP over 5 years as an achievement indicator, and will distinguish between the prefinancial-crisis period (2003 to 2007) and post-crisis period (2008 to 2012). I will also normalise the values with respect to the average, since the analytical results presented in Section 3 refer to equilibrium levels of $y$ and $h e$. In this analysis, I eliminated 38 of 218 countries in the World Bank Development Indicators dataset that lacked data on LEB or per capita GDP data in both periods. Where only one of the two values was missing, I used the value from the other period to provide the missing data.

To obtain the predicted $h e^{*}$ and $y^{*}$ that were compared with the observed LEB and per capita GDP, I used the following proxies: $a y=1 /(1-$ Gini), where Gini represents the value of the Gini coefficient; $e d=$ the enrolment in tertiary school ( $\%$ of the students), with $-1+$ each value divided by the mean to obtain a normalized value; $o c=-\%$ industry employment; and $e m=1-\%$ unemployed with $-1+$ each value divided by the mean to obtain a normalized value. In this calculation, I used Gini coefficients from the CIA World Fact Book when the coefficients were missing from the World Bank database. I eliminated 50 countries due to lack of data on tertiary education or industry employment, and 23 countries due to lack of data on Gini coefficients.

Table $\mathrm{S}$ in Supplementary Materials shows the calculated $f r$ values for each country, which make the predicted health $h e^{*}$ consistent with the observed LEB for given values of per capita GDP, ay, $e d, o c$, and $e m$.

As expected, apart from the atheist KOR and the former English colonies QAT and IRL, significantly negative $f r$ (i.e., $f r<-1$ in both periods) were obtained for Protestant DCs (AUS, CHE, DNK, FIN, IRL, ISL, LUX, NLD, NOR, SWE, and USA) in both periods. In contrast, a negative $f r$ was observed only in the first period in BEL, GBR, GRC, and KWT, and only in the second period in AUT, CHE, HKG, and NZL.

To obtain insights on the relative proportions of the population who adopted the $\alpha, \beta$, and $\gamma$ attitudes, I used the calculated $f r$ to compare the observed per capita GDP $\left(y^{\circ}\right)$ and calculated the equilibrium income $\left(y^{*}\right)$ at different pairs of extreme values for $\alpha$ and $\gamma$ (i.e., $\alpha=0$ and $\gamma=0, \alpha=1$ and $\gamma=0, \alpha=0$ and $\gamma=1, \alpha=1$ and $\gamma=1$ ). To do so, I relied on the negative impacts of both $\alpha$ and $\gamma$ on the estimated income (see Appendix for details). In particular, if $y^{*}-y^{\circ}>0$ at $\alpha=0$ and $\gamma=0$, then both $\alpha$ and $\gamma$ must increase to reduce the overestimate of $y^{*}$; if $y^{*}-y^{\circ}>0$ at $\alpha=1$ and $\gamma=0$, then $\gamma$ must increase to reduce the overestimate of $y^{*}$; if $y^{*}-y^{\circ}>0$ at $\alpha=0$ and $\gamma=1$, then $\alpha$ must increase to reduce the overestimate of $y^{*}$; and if $y^{*}-y^{\circ}<0$ at $\alpha=1$ and $\gamma=1$, then both $\alpha$ and $\gamma$ must decrease to increase the underestimate of $y^{*}$. Table $\mathrm{S}$ in Supplementary Materials shows the calculated differences between the observed per capita GDP and the calculated income (i.e., $y^{*}-y^{\circ}$ ). Table 3 summarises the description of the countries as having either single dominant or co-dominant attitude types. 
Table 3.Estimated attitudes as a function of a country's social characteristics (religion and education); * means “an attitude suggested by a religion"; $\neg$ means "different from"; $\approx$ means "similar to". Latin American (LA) countries: ARG, BLZ, BOL, CRI, ECU, GTM, HND, PAN, PER, PRY, URY, and VEN. Mid-educated ex-SU (former members of the Soviet Union with middle educational levels): BGR, ROU, and SVN. High-educated exSU (former members of the Soviet Union with high educational levels): CZE, EST, HUN, LTU, LVA, and RUS. Low Educated Muslim (Muslim countries with a low educational level): AZE, BFA, BGD, IDN, MAR, MDA, PAK, PHL, TZA, and UGA. Mid-educated Muslim (Muslim countries with a middle educational level): ALB, GEO, JOR, KGZ, MLI, SEN, SRB, TJK, and YEM. High-educated Muslim (Muslim countries with a high educational level): ARM, BIH, DZA, EGY, IRQ, MKD, MNE, and TUN.

\begin{tabular}{|c|c|c|c|c|}
\hline & \multicolumn{2}{|c|}{ DC } & \multicolumn{2}{|r|}{ LDC } \\
\hline & Income-equal & Income-unequal & Income-equal & Income-unequal \\
\hline Protestant & $\begin{array}{c}\beta \gamma(\text { AUS, LUX, } \\
\text { NLD, SWE) }\end{array}$ & $\begin{array}{c}\beta^{*}(\mathrm{CHE}, \mathrm{DNK}, \mathrm{FIN}, \\
\text { HKG, ISL, NZL USA) }\end{array}$ & & \\
\hline $\begin{array}{l}\text { Non-Protestant } \\
\text { Christian }\end{array}$ & $\begin{array}{c}\gamma^{*}(\mathrm{IRL}, \mathrm{BEL}, \mathrm{GBR}, \\
\text { GRC, UKR, AUT) }\end{array}$ & $\begin{array}{c}\gamma \beta \text { (CHL, FRA, ITA, } \\
\text { POL, SVK) }\end{array}$ & & $\begin{array}{c}\neg \gamma \text { (Mid-educated ex-SU, } \\
\text { LA, ETH) }\end{array}$ \\
\hline Muslim & & & $\begin{array}{c}\alpha \gamma(\text { High- } \\
\text { educated), } \approx \alpha \gamma \\
\text { (TUR) }\end{array}$ & $\begin{array}{c}\alpha^{*}(\text { Low-educated }), \neg \alpha \\
\text { (Mid-educated), } \approx \alpha(\text { IRN })\end{array}$ \\
\hline Jewish & & $\beta^{*}$ (ISR) & & \\
\hline Hindu & & & $\gamma^{*}$ & $\approx \gamma($ IND $)$ \\
\hline Buddhist & & & $(\alpha \beta)^{*}$ & $\begin{array}{c}\neg \gamma(\mathrm{BTN}, \mathrm{KHM}, \mathrm{LKA}, \\
\text { THA, VNM })\end{array}$ \\
\hline Atheist & $\beta^{*}(\mathrm{CZE}, \mathrm{HVR})$ & & $\begin{array}{c}\beta^{*} \text { (High-educated } \\
\text { ex-SU) }\end{array}$ & $\beta^{*}(\mathrm{KOR})$ \\
\hline
\end{tabular}

These results are consistent with predictions if religions account for the prevailing attitudes, with the following constancies: $\alpha$ attitudes are dominant in Muslim LDCs, with larger $\gamma$ shares in more educated countries; a prevalence of $\beta$ attitudes in Protestant DCs, with larger $\gamma$ shares in less income-unequal countries; a prevalence of $\gamma$ attitudes in non-Protestant Christian DCs, with larger $\beta$ shares in more income-unequal countries; and a prevalence of $\beta$ attitudes in more educated atheist and Jewish countries.

Indeed, the Muslim maxim "That which is left by Allah for you (after accounting for the rights of the people) is better for you" (Surat Hud, Ayah 86) seems to evoke an $\alpha$ attitude (see also Hamidi et al., 2010); the Protestant maxim that "Vocation from God is not limited to clergy or church, but it can be applied to any occupation or trade" (Luther, 1520) suggests a $\beta$ attitude (see also van Hoorn and Maseland, 2013); the Non-Protestant Christian maxim that "Only in God will one find the truth and happiness one never stops searching for" (St. Augustine, 401) seems to evoke a $\gamma$ attitude (see also Vayalilkarottu, 2012); and the many Jewish commandments concerning the kashrut (fitness) of one's money in the Torah, which are expanded upon in the Mishnah and the Talmud, suggest a $\beta$ attitude (see also Van Praag et al., 2010).

The following additional insights were obtained from my analysis. First, movements towards a modern (i.e., market oriented) society implied an increase in $\beta$, both in atheist and Non-Protestant Christian societies. Some of these societies have firmly moved to $\beta$ (CYP, CZE, MEX, MLT, and PRT), some are moving in this direction (CHL, FRA, HVR, ITA, POL, and SVK), and some have not yet completed this movement (ESP). Second, the economic crisis reduced $\beta$ (HKG and ISL) and increased $\gamma$ (CHE, DNK, FIN, and USA) in Protestant societies, whereas poor and income-unequal societies adopted an $\alpha$ attitude, both for Non-Protestant Christian countries (BWA, CMR, COL, JAM, MDA, MNG, and NIC) and Protestant countries (GHA, MDG, and NAM). Third, Buddhist countries could not be simply characterised, since this religion cannot be linked to any single typified attitude ( $\alpha$ or $\beta$ or $\gamma$ ), but rather combined aspects of the $\alpha$ and $\beta$ attitudes; BTN, KHM, LKA, THA, and VNM could be better represented as a non- $\gamma$ group. Fourth, attitudes could not be characterised when many religions had high representation in a country: DEU (30\% Non-Protestant Christian, 30\% Protestant, 20\% atheist), CMR (38\% Catholic, 15\% Protestant Christian, 22\% Muslim), MDG (22\% Catholic, 22\% Protestant, 50\% local religions), MUS (49\% Hindu, 32\% 
Protestant, 17\% Muslim), QAT (77\% Muslim, 12\% Hindu, 10\% Protestant), and CHN (57\% Taoist, $25 \%$ atheist, $14 \%$ Buddhist).

Therefore, attitudes for a representative individual at a country level seem to be accounted for by the most popular religion combined with the observed education and inequality levels when certain short-run dynamics (e.g., a crisis) or long-term dynamics (e.g., market orientations) hold.

\section{Discussion}

In this paper, I developed an original analytical model that accounts for three main attitudes towards life (i.e., $\alpha, \beta$, and $\gamma$ for Aristotelian, Epicurean, and Stoic attitudes, respectively). The model accounts for all variables that have been shown by empirical and theoretical studies to affect health and happiness at an individual level for a general population (i.e., employment, occupation type, education, ethical freedom, opportunity distribution), and considers three main approaches (i.e., exante behavioural or statistical, ex-post behavioural).

The model permitted an empirical test based on reasonable assumptions such as compensation for individual lucky and unlucky events at a country level, and the prevalence of equilibrium attitudes in the long-run.

The model can explain the results of some empirical studies. For example, Bjørnskov (2008) found an inverse relationship between happiness $(h a)$ and longevity (he) at the country level. The present model can explain this result by considering the impacts of ethical freedom on health $(\partial h e / \partial f r>0)$ and happiness $(\partial h a / \partial f r<0)$, and by assuming that the latter effect is larger than the former; that is, $\partial h e / \partial f r+\partial h a / \partial f r<0$. Blanchflower \& Oswald (2008, 2009) found a U-shaped curve for psychological well-being (he, based on psychiatric assessment scores, mental health levels, and depression and anxiety levels) over an individual's life cycle, with the minimum score in middle age. The present model can explain this result by remembering the impacts of attitudes on health (i.e., $\partial$ he/ $\partial \beta>\partial h e / \partial \alpha>\partial h e / \partial \gamma>0$ ), and by assuming that a $\beta$ attitude prevails in young people (because the $\delta$ value is close to 0 ), a $\gamma$ attitude prevails in middle-aged people, and an $\alpha$ attitude prevails in old people.

The model can confirm the insights obtained from some theoretical studies. For example, Zagonari (2009) found higher and lower happiness levels in Protestant and non-Protestant Christian countries, respectively. This can be replicated with the current model by stressing that in the twoequation dynamic model (i.e., dhe $=\partial h e / \partial f r d f r+\partial h e / \partial y d y+d h a, d h a=\alpha \partial h a / \partial y d y-\alpha$ dha/ $f r d f r+\beta$ $d y+\gamma d y+d h e)$ the health level could be fixed (i.e., dhe $=0)$ rather than the happiness level $(d h a=0)$, and by assuming that the impact of ethical freedom on health ( $\partial h e / \partial f r>0)$ is smaller than the impact of ethical freedom on happiness for the $\alpha$ attitude $(-\alpha \partial h a / \partial f r>0)$ (i.e., $\partial h e / \partial f r-\alpha \partial h a / \partial f r<0)$.

\section{Conclusions}

The analytical approach adopted in the present study successfully accounted for the effects of culture on happiness and health by emphasising measurable individual characteristics (i.e., education, employment, occupation) as well as measurable social characteristics (ethical freedom, equity in achievements).

However, some caveats concerning the results obtained in this paper need to be highlighted. I normalised $h a^{*}$ to 1 in order to obtain an analytical solution. However, the present analysis showed that the relationship between happiness, health, and ethical freedom was consistent with the relationship between happiness and ethical freedom discussed in Zagonari (2009). I tested the robustness of the analytical model by assuming that people are neither lucky nor unlucky on average, and by applying the average values at $u^{*}=0$. Alternatively, one could estimate a dynamic econometric model with cross-country time-series individual data with error values that could potentially characterise each individual. However, few countries and even fewer cultures present these kinds of datasets. I disregarded a possible preference for large variability in the short-run achievements over a large mean in the long-run achievement by comparing happiness, health, and achievements in the long-run. Alternatively, one could account for short-term fluctuations in 
choosing their attitude. I tested the robustness of the analytical model by presenting qualitative insights related to $f r, \alpha, \beta$, and $\gamma$. Alternatively, one could obtain an econometric estimation of these parameters using cross-country individual data. However, few countries and even fewer cultures present these kinds of datasets. I disregarded the potential for remorse in the ex-post behavioural approach. Alternatively, one could account for regret in choosing their attitude. I tested the robustness of the analytical model by assuming that people are observed at a long-run equilibrium and by looking for insights into $\alpha, \beta$, and $\gamma$ at this equilibrium. Alternatively, one could estimate a dynamic econometric model with cross-country time-series individual data and $\alpha, \beta$, and $\gamma$ values that change over time. However, few countries and even fewer cultures present these kinds of datasets.

The main future developments for the framework suggested in this paper are as follows. It may be worth estimating coefficients to be attached to the model variables from alternative cultural contexts such as Russia (Graham et al., 2004), Peru (Izquierdo, 2005), Sweden (Andersson, 2008), Japan (Oshio \& Kobayashi, 2010) and the United States (Borgonovi, 2008), possibly by relying on a cross-country time-series dataset. It may be worth characterising the ethical and religious traditions in terms of typified attitudes, for example, by emphasising combinations of $\alpha$ and $\beta$ attitudes in Buddhism (Bilimoria et al., 2008; Tomer, 2011).

\section{Appendix}

If $\gamma=1$, under the assumptions that $u=0, f y=f h=0$, and $h a^{*}=0$ to depict the normalised situations at a country level, $\partial y^{*} / \partial \alpha=f r(e m+e d-1+3 f r) /(\alpha+2 f r)^{2}$, where $\partial y^{*} / \partial \alpha>0$ if $f r>0$ and $f r>(1-e d-e m) / 3$ or if $f r<0$ and $f r<(1-e d-e m) / 3$. These conditions are unlikely to be met, because they require the prevalence of features characterizing Protestant DCs (e.g., negative $f r$ ) combined with features characterizing LDCs (e.g., small $e d$ and $e m$ ). We can assume that $y^{*}$ negatively depends on $\alpha$. If $\alpha=1$, under the assumptions that $u=0, f y=f h=0$, and $h a^{*}=0$ to depict the normalised situations at a country level, $\partial y^{*} / \partial \gamma=f r[1-f r+f r(1+e m+e d+f r)] /(1+f r+\gamma f r)^{2}$, where $\partial y^{*} / \partial \gamma>0$ if $f r>0$ and $f r>(1 / 2)$ $\left\{-e d-e m+\operatorname{Sqrt}\left[(e m+e d)^{2}-4\right]\right\}$ or if $f r<0$ and $f r<(1 / 2)\left\{-e d-e m-\operatorname{Sqrt}\left[(e m+e d)^{2}-4\right]\right\}$. These conditions are unlikely to be met, because they require the prevalence of features characterizing Protestant DCs (e.g., negative $f r$ ) combined with features characterizing LDCs (e.g., small ed and em). We can assume that $y^{*}$ negatively depends on $\gamma$. Note that these results are reasonable, since it is likely that, on average, $y^{*}$ is larger if a $\beta$ attitude prevails.

\section{References}

Andersson, P. (2008) Happiness and health: Well-being among the self-employed, The Journal of Socio-Economics 37:213-236

Becchetti, L., Rossetti, F. (2009) When money does not buy happiness: The case of "frustrated achievers, The Journal of Socio-Economics 38:159-167

Bilimoria, P. et al. (2008) Ethical Studies, Overview (Eastern), Encyclopaedia of Violence, Peace, \& Conflict (Second Edition): 720-739

Binder, M., Coad, A. (2010) An examination of the dynamics of well-being and life events using vector autoregressions, Journal of Economic Behavior \& Organization 76:352-371

Binder, M., Coad, A. (2011) From Average Joe's happiness to Miserable Jane and Cheerful John: using quantile regressions to analyze the full subjective well-being distribution, Journal of Economic Behavior \& Organization 79:275-290

Binder, M., Coad, A. (2013) "I'm afraid I have bad news for you..." Estimating the impact of different health impairments on subjective well-being, Social Science \& Medicine 87:155-167

Bjørnskov, C. (2008) Healthy and happy in Europe? On the association between happiness and life expectancy over time, Social Science \& Medicine 66:1750-1759

Blanchflower, D.G., Oswald, A.J. (2008) Hypertension and happiness across nations, Journal of Health Economics 27:218-233 
Blanchflower, D.G., Oswald, A.J. (2009) The U-shape without controls: A response to Glenn, Social Science \& Medicine 69:486-488

Blanchflower, D.G., Oswald, A.J.(2008) Well-being U-shaped over the life cycle?, Social Science \& Medicine 66:1733-1749

Borgonovi, F. (2008) Doing well by doing good. The relationship between formal volunteering and self-reported health and happiness, Social Science \& Medicine 66:2321-2334

Bostani, M., Saiiari, A. (2011) Comparison Emotional Intelligence and Mental Health between Athletic and Non-Athletic Students, Procedia-Social and Behavioral Sciences 30:2259-2263

Brown, P.H., Tierney, B. (2009) Religion and subjective well-being among the elderly in China, The Journal of Socio-Economics 38:310-319

Carlsson, F. et al. (2014) Subjective well-being among preadolescents and their parents-Evidence of intergenerational transmission of well-being from urban China, The Journal of Socio-Economics 48:11-18

Diener, E., Chan, M.Y. (2011) Happy People Live Longer: Subjective Well-Being: Contributes to Health and Longevity, Applied Psychology: Health And Well-Being 3:1-43

Easterlin, R.A. (2009) Lost in transition: Life satisfaction on the road to capitalism, Journal of Economic Behavior \& Organization 71:130-145

Gardner, J., Oswald, A.J. (2007) Money and mental wellbeing: A longitudinal study of mediumsized lottery wins, Journal of Health Economics 26:49-60

Gatab, T.A., Pirhayti, S. (2012) The Effect of the Selected Exercise on Male Students' Happiness and Mental Health, Procedia-Social and Behavioral Sciences 46:2702-2705

Gatab, T.A. et al. (2011) The Relationship Between Psychological Health, Happiness and Life Quality in the Students, Procedia-Social and Behavioral Sciences 30:1983-1985

Golden, L., Wiens-Tuers, B. (2006) To your happiness? Extra hours of labor supply and worker well-being, The Journal of Socio-Economics 35:382-397

Graham, C. et al. (2004) Does happiness pay?: An exploration based on panel data from Russia, Journal of Economic Behavior \& Organization 55:319-342

Guney, S. (2011) The Positive Psychotherapy Inventory (PPTI): Reliability and Validity Study in Turkh Population, Procedia-Social and Behavioral Sciences 29:81-86

Haji,T. M. et al. (2011) The Effectiveness of Life Skills Training on Happiness, Quality of Life and Emotion Regulation, Procedia -Social and Behavioral Sciences 30:407-411

Hamidi, F. et al. (2010) The Role of Islamic Education in Mental Health, Procedia-Social and Behavioral Sciences 5:1991-1996

Izquierdo, C. (2005) When "health" not enough: societal, individual and biomedical assessments of well-being among the Matsigenka of the Peruvian Amazon, Social Science \& Medicine 61:767-783 Kiyani, R. et al. (2011) The Survey Compares Mental Health and Happiness of Athlete and NonAthlete Employed People, Procedia-Social and Behavioral Sciences 30:1894-1896

Lelkes, O. (2006) Knowing what is good for you: Empirical analysis of personal preferences and the "objective good", The Journal of Socio-Economics 35:285-307

Maselko, J., Kubzansky, L.D. (2006) Gender differences in religious practices, spiritual experiences and health: Results from the US General Social Survey, Social Science \& Medicine 62:2848-2860

Mukuria, C., Brazier, J. (2013) Valuing the EQ-5D and the SF-6D health states using subjective well-being: A secondary analysis of patient data, Social Science \& Medicine 77:97-105

Oshio, T., Kobayashi, M. (2010) Income inequality, perceived happiness, and self-rated health: Evidence from nationwide surveys in Japan, Social Science \& Medicine 70:1358-1366

Pedersen, P.J., Schmidt, T.D. (2011) Happiness in Europe: Cross-country differences in the determinants of satisfaction with main activity, The Journal of Socio-Economics 40:480-489

Peiró, A. (2006) Happiness, satisfaction and socio-economic conditions: Some international evidence, The Journal of Socio-Economics 35:348-365

Powdthavee, N., van den Berg,B. (2011) Putting different price tags on the same health condition: Re-evaluating the well-being valuation approach, Journal of Health Economics 30:1032-1043 
Salary, S., Shaieri, M.R. (2013) Study of the Relationship between Happiness and Dimensions of Psychos, Neuros and Personality Extraversion, Procedia-Social and Behavioral Sciences 84:11431148

Schatz, E. et al. (2012) The impact of pensions on health and wellbeing in rural South Africa: Does gender matter?, Social Science \& Medicine 75:1864-1873

Shayan, N., Gatab, T. (2012) The Effectiveness of Social Skills Training on Students' Levels of Happiness, Procedia-Social and Behavioral Sciences 46:2693-2696

Tomer, J.F. (2011) Enduring happiness: integrating the hedonic and eudaimonic approaches, Journal of Socio-Economics 40:530-537

van Campen, C., Cardol, M. (2009) When work and satisfaction with life do not go hand in hand: Health barriers and personal resources in the participation of people with chronic physical disabilities, Social Science \& Medicine 69:56-60

van Hoorn, A., Maseland, R. (2013) Does a Protestant work ethic exists? Evidence from the wellbeing effect of unemployment, Journal of Economic Behavior \& Organization 91:1-12

Van Praag, B.M.S. et al. (2010) Happiness and financial satisfaction in Israel: Effects of religiosity, ethnicity, and war, Journal of Economic Psychology 31:1008-1020

Vayalilkarottu, J. (2012) Holistic health and well-being: A psycho-spiritual/(religious and theological perspective, Asian Journal of Psychiatry 5:347-350

Verme, P. (2009) Happiness, freedom and control, Journal of Economic Behavior \& Organization 71:146-161

Yahyaei, D. et al. (2012) The School Role in Lively Education and Student's Mental Health, Procedia-Social and Behavioral Sciences 47:1408-1412

Yakovlev, P., Leguizamon, S. (2012) Ignorance not bless: On the role of education in subjective well-being, The Journal of Socio-Economics 41:806-815

Zagonari, F. (2009) Which ethics will make us individually and socially happier? A cross-culture and cross-development analytical model, Journal of Happiness Studies 12:77-103

\section{Supplementary Materials}

Table S. Estimated $f r$ and differences between the estimated and observed per capita GDP for different extreme values of attitudes $\alpha$ and $\gamma$. Country codes are from the World Bank Development Indicators dataset.

$$
\begin{array}{cccccccccc}
f r & f r & \alpha=0 & \alpha=0 & \alpha=1 & \alpha=1 & \alpha=0 & \alpha=0 & \alpha=1 & \alpha=1 \\
& & \gamma=0 & \gamma=0 & \gamma=0 & \gamma=0 & \gamma=1 & \gamma=1 & \gamma=1 & \gamma=1 \\
\text { 2003-07 } & 2008-12 & 2003-07 & 2008-12 & 2003-07 & 2008-12 & 2003-07 & 2008-12 & 2003-07 & 2008-12
\end{array}
$$

$\begin{array}{lccccccccccc}\begin{array}{c}\text { Country code } \\ \text { AFG }\end{array} & & & & & & & & & & & \\ \text { AGO } & & & & & & & & & & \\ \text { ALB } & 2.13 & 1.65 & -0.94 & -0.89 & 0.20 & 0.22 & 0.26 & 0.24 & 0.74 & 0.69 \\ \text { ARE } & & & & & & & & & & \\ \text { ARG } & 0.56 & 0.75 & -1.33 & -1.62 & -0.02 & -0.09 & -0.13 & -0.24 & 0.30 & 0.33 \\ \text { ARM } & 1.75 & 1.46 & 0.72 & -0.30 & 1.33 & 0.61 & 1.22 & 0.60 & 1.49 & 0.95 \\ \text { ATG } & 1.35 & 1.53 & & & & & & & & \\ \text { AUS } & -1.42 & -1.43 & -2.09 & -1.97 & 0.33 & 0.84 & -1.38 & -1.34 & -0.36 & -0.28 \\ \text { AUT } & -0.64 & -1.00 & -2.27 & -2.12 & -2.82 & 146.11 & -1.54 & -1.44 & 2.11 & -0.05 \\ \text { AZE } & 2.35 & 2.05 & -1.45 & -1.61 & -0.14 & -0.30 & 0.03 & -0.17 & 0.57 & 0.37 \\ \text { BDI } & & & & & & & & & & \\ \text { BEL } & -1.04 & -0.91 & -1.81 & -1.70 & 21.75 & -9.93 & -1.23 & -1.12 & 0.25 & 0.69 \\ \text { BEN } & 2.75 & 2.59 & & & & & & & & \\ \text { BFA } & 2.68 & 2.64 & -1.54 & -1.53 & -0.14 & -0.13 & 0.10 & 0.11 & 0.70 & 0.71 \\ \text { BGD } & 2.99 & 2.83 & -1.83 & -1.67 & -0.39 & -0.26 & -0.02 & 0.05 & 0.57 & 0.64\end{array}$




\begin{tabular}{|c|c|c|c|c|c|c|c|c|c|c|}
\hline BGR & 1.49 & 1.06 & -1.67 & -1.59 & -0.33 & -0.30 & -0.32 & -0.34 & 0.24 & 0.16 \\
\hline BHR & 0.75 & 1.08 & & & & & & & & \\
\hline \multicolumn{11}{|l|}{ BHS } \\
\hline BIH & 2.30 & 1.98 & 0.60 & 0.37 & 1.26 & 1.04 & 1.09 & 0.93 & 1.42 & 1.24 \\
\hline BLR & 0.83 & 0.38 & -2.09 & -1.91 & -0.34 & -0.20 & -0.42 & -0.44 & 0.17 & 0.07 \\
\hline BLZ & 2.33 & 2.19 & & & & & & & & \\
\hline BOL & 1.77 & 1.86 & -1.82 & -1.94 & -0.27 & -0.37 & -0.25 & -0.32 & 0.46 & 0.39 \\
\hline \multicolumn{11}{|l|}{ BRA } \\
\hline BRB & -0.33 & -0.22 & & & & & & & & \\
\hline \multicolumn{11}{|l|}{$\mathrm{BRN}$} \\
\hline BTN & 2.67 & 2.51 & -1.83 & -1.74 & -0.41 & -0.35 & -0.15 & -0.13 & 0.46 & 0.46 \\
\hline BWA & 1.92 & 1.80 & 0.19 & 0.37 & 0.80 & 0.89 & 0.82 & 0.93 & 1.12 & 1.17 \\
\hline \multicolumn{11}{|l|}{ CAF } \\
\hline \multicolumn{11}{|l|}{ CAN } \\
\hline CHE & -0.98 & -1.29 & -2.27 & -2.09 & -49.45 & 3.37 & -1.70 & -1.70 & -0.04 & -0.36 \\
\hline CHL & 1.00 & 0.47 & -1.78 & -1.65 & -0.44 & -0.40 & -0.52 & -0.51 & 0.02 & -0.11 \\
\hline $\mathrm{CHN}$ & 2.67 & 2.42 & -2.16 & -2.10 & -0.66 & -0.64 & -0.42 & -0.46 & 0.26 & 0.21 \\
\hline \multicolumn{11}{|l|}{ CIV } \\
\hline CMR & 2.72 & 2.56 & -1.67 & -1.56 & -0.26 & -0.16 & -0.04 & 0.03 & 0.60 & 0.66 \\
\hline $\mathrm{COG}$ & 2.85 & 2.66 & & & & & & & & \\
\hline $\mathrm{COL}$ & 1.90 & 1.62 & -1.09 & -1.11 & 0.08 & 0.07 & 0.08 & 0.05 & 0.64 & 0.60 \\
\hline \multicolumn{11}{|l|}{$\mathrm{COM}$} \\
\hline \multicolumn{11}{|l|}{$\mathrm{CPV}$} \\
\hline CRI & 2.02 & 1.42 & -1.97 & -1.68 & -0.57 & -0.32 & -0.49 & -0.34 & 0.14 & 0.25 \\
\hline CYP & 0.48 & 0.03 & -2.17 & -1.71 & -1.08 & -1.11 & -1.11 & -0.91 & -0.82 & -1.09 \\
\hline $\mathrm{CZE}$ & 0.63 & 0.27 & -2.19 & -2.06 & -0.94 & -0.82 & -1.00 & -0.92 & -0.61 & -0.64 \\
\hline DEU & -0.66 & -0.56 & -1.73 & -1.89 & -3.23 & -2.50 & -1.15 & -1.23 & 2.79 & 8.87 \\
\hline \multicolumn{11}{|l|}{ DJI } \\
\hline \multicolumn{11}{|l|}{ DMA } \\
\hline DNK & -1.67 & -1.38 & -2.14 & -1.76 & -0.19 & 1.81 & -1.46 & -1.25 & -0.59 & -0.16 \\
\hline DOM & 1.89 & 1.81 & -0.66 & -0.77 & 0.39 & 0.28 & 0.39 & 0.29 & 0.86 & 0.76 \\
\hline DZA & 2.36 & 2.17 & -0.69 & -1.38 & 0.35 & -0.11 & 0.37 & 0.00 & 0.82 & 0.53 \\
\hline ECU & 1.61 & 1.70 & -1.57 & -1.79 & -0.19 & -0.35 & -0.17 & -0.31 & 0.42 & 0.32 \\
\hline EGY & 2.06 & 2.10 & -1.34 & -1.25 & -0.03 & 0.02 & 0.10 & 0.13 & 0.63 & 0.65 \\
\hline \multicolumn{11}{|l|}{ ERI } \\
\hline ESP & -0.70 & -0.77 & -1.77 & -0.39 & -2.55 & -7.07 & -1.02 & -0.23 & 2.06 & 3.63 \\
\hline EST & 0.15 & 0.19 & -1.95 & -1.30 & -0.47 & -0.44 & -0.69 & -0.38 & -0.35 & -0.31 \\
\hline ETH & 2.83 & 2.72 & -0.28 & -0.24 & 0.78 & 0.81 & 0.83 & 0.84 & 1.29 & 1.30 \\
\hline FIN & -1.92 & -1.65 & -1.83 & -1.69 & 0.00 & 0.58 & -1.19 & -1.09 & -0.35 & -0.16 \\
\hline \multicolumn{11}{|l|}{ FJI } \\
\hline FRA & -0.55 & -0.37 & -1.74 & -1.53 & -2.29 & -1.83 & -1.07 & -0.92 & 39.11 & -3.53 \\
\hline \multicolumn{11}{|l|}{ FSM } \\
\hline \multicolumn{11}{|l|}{ GAB } \\
\hline GBR & -1.08 & -0.66 & -2.11 & -1.66 & 7.00 & -2.96 & -1.38 & -1.01 & 0.18 & 5.50 \\
\hline GEO & 1.58 & 2.03 & -0.84 & -0.39 & 0.37 & 0.63 & 0.38 & 0.63 & 0.90 & 1.09 \\
\hline GHA & 2.87 & 2.73 & -1.76 & -1.66 & -0.33 & -0.25 & -0.03 & 0.02 & 0.59 & 0.63 \\
\hline \multicolumn{11}{|l|}{ GIN } \\
\hline GMB & & & & & & & & & & \\
\hline
\end{tabular}




\begin{tabular}{|c|c|c|c|c|c|c|c|c|c|c|}
\hline \multicolumn{11}{|l|}{ GNB } \\
\hline GRC & -1.28 & -0.83 & -1.61 & -0.86 & 0.97 & -5.92 & -0.82 & -0.33 & 0.27 & 2.13 \\
\hline \multicolumn{11}{|l|}{ GRD } \\
\hline GTM & 2.56 & 2.54 & -2.22 & -1.97 & -0.69 & -0.51 & -0.53 & -0.36 & 0.21 & 0.35 \\
\hline \multicolumn{11}{|l|}{ GUY } \\
\hline HKG & -0.58 & -1.46 & -1.90 & -1.95 & -3.51 & 1.81 & -1.31 & -1.42 & -19.86 & 0.26 \\
\hline HND & 2.69 & 2.57 & -2.06 & -1.96 & -0.57 & -0.48 & -0.42 & -0.36 & 0.32 & 0.38 \\
\hline HRV & 0.98 & 0.70 & -1.41 & -1.26 & -0.35 & -0.31 & -0.36 & -0.30 & 0.01 & -0.02 \\
\hline \multicolumn{11}{|l|}{ HTI } \\
\hline HUN & 0.29 & 0.43 & -2.04 & -1.45 & -0.55 & -0.43 & -0.73 & -0.43 & -0.34 & -0.19 \\
\hline IDN & 2.57 & 2.36 & -1.31 & -1.49 & -0.02 & -0.13 & 0.16 & 0.05 & 0.72 & 0.62 \\
\hline IND & 2.78 & 2.60 & -1.87 & -1.95 & -0.43 & -0.47 & -0.11 & -0.18 & 0.50 & 0.44 \\
\hline IRL & -1.39 & -1.26 & -2.27 & -1.11 & 1.22 & 6.63 & -1.72 & -0.92 & -0.51 & 0.72 \\
\hline IRN & 2.18 & 1.59 & -1.53 & -1.42 & -0.29 & -0.16 & -0.21 & -0.14 & 0.35 & 0.39 \\
\hline IRQ & 2.67 & 2.61 & 0.06 & -0.55 & 0.98 & 0.53 & 0.92 & 0.59 & 1.34 & 1.05 \\
\hline ISL & -1.47 & -1.23 & -2.35 & -1.81 & -0.35 & 2.37 & -1.58 & -1.16 & -0.65 & 0.01 \\
\hline ISR & -0.21 & -0.15 & -1.67 & -1.79 & -1.02 & -0.96 & -0.79 & -0.86 & -1.37 & -1.17 \\
\hline ITA & -0.63 & -0.39 & -2.04 & -1.72 & -1.98 & -1.54 & -1.20 & -0.95 & 3.00 & -3.47 \\
\hline JAM & 2.16 & 2.12 & -1.27 & -0.94 & -0.05 & 0.21 & 0.02 & 0.25 & 0.56 & 0.77 \\
\hline JOR & 1.87 & 1.77 & -1.02 & -0.93 & 0.21 & 0.26 & 0.27 & 0.30 & 0.79 & 0.80 \\
\hline JPN & -0.52 & -0.30 & -2.34 & -2.13 & -1.69 & -1.35 & -1.41 & -1.20 & -13.19 & -2.05 \\
\hline KAZ & 0.97 & 1.26 & -1.47 & -1.63 & -0.09 & -0.28 & -0.11 & -0.25 & 0.36 & 0.24 \\
\hline KEN & 2.74 & 2.74 & & & & & & & & \\
\hline KGZ & 1.84 & 1.95 & -1.44 & -1.42 & 0.01 & 0.01 & 0.16 & 0.17 & 0.73 & 0.73 \\
\hline KHM & 3.05 & 2.77 & -2.26 & -2.22 & -0.74 & -0.67 & -0.31 & -0.30 & 0.34 & 0.36 \\
\hline \multicolumn{11}{|l|}{ KIR } \\
\hline KOR & -1.29 & -1.34 & -2.35 & -2.11 & -2.95 & -1.62 & -1.15 & -1.03 & -0.34 & -0.12 \\
\hline KWT & -1.06 & -0.38 & & & & & & & & \\
\hline \multicolumn{11}{|l|}{ LAO } \\
\hline LBN & 1.30 & 1.23 & & & & & & & & \\
\hline \multicolumn{11}{|l|}{ LBR } \\
\hline \multicolumn{11}{|l|}{ LBY } \\
\hline LCA & 2.27 & 2.18 & & & & & & & & \\
\hline LKA & 2.84 & 2.67 & -1.82 & -1.89 & -0.42 & -0.47 & -0.21 & -0.28 & 0.44 & 0.38 \\
\hline \multicolumn{11}{|l|}{ LSO } \\
\hline LTU & -0.03 & -0.18 & -1.76 & -1.00 & -0.19 & -0.46 & -0.50 & -0.17 & -0.22 & -0.74 \\
\hline LUX & -2.67 & -2.74 & -2.06 & -1.82 & 1.20 & 1.41 & -2.92 & -2.73 & -1.83 & -1.65 \\
\hline LVA & -0.04 & 0.14 & -1.72 & -0.79 & -0.08 & -0.20 & -0.44 & 0.00 & -0.13 & -0.09 \\
\hline MAC & -1.71 & -2.82 & -2.21 & -2.12 & 0.17 & 0.20 & -1.66 & -2.22 & -0.63 & -1.25 \\
\hline MAR & 2.78 & 2.66 & -1.28 & -1.34 & -0.02 & -0.06 & 0.15 & 0.10 & 0.71 & 0.67 \\
\hline MDA & 1.94 & 2.02 & -1.58 & -1.63 & -0.11 & -0.16 & 0.04 & 0.00 & 0.64 & 0.61 \\
\hline MDG & 2.85 & 2.83 & -1.59 & -1.56 & -0.20 & -0.17 & 0.03 & 0.04 & 0.67 & 0.69 \\
\hline MDV & 2.94 & 2.59 & & & & & & & & \\
\hline MEX & 1.90 & 1.76 & -2.30 & -1.99 & -0.85 & -0.65 & -0.77 & -0.61 & -0.13 & -0.01 \\
\hline MKD & 2.18 & 1.73 & 1.23 & 1.07 & 1.64 & 1.40 & 1.44 & 1.29 & 1.67 & 1.46 \\
\hline MLI & 2.75 & 2.70 & -1.03 & -0.95 & 0.23 & 0.28 & 0.39 & 0.43 & 0.94 & 0.98 \\
\hline MLT & 1.08 & 0.75 & -2.00 & -1.84 & -0.88 & -0.84 & -0.88 & -0.84 & -0.48 & -0.53 \\
\hline
\end{tabular}




\begin{tabular}{|c|c|c|c|c|c|c|c|c|c|c|}
\hline MNE & 1.95 & 1.05 & 0.24 & -0.24 & 0.90 & 0.42 & 0.74 & 0.42 & 1.04 & 0.64 \\
\hline MNG & 1.57 & 1.38 & -1.49 & -1.59 & -0.01 & -0.04 & 0.07 & 0.00 & 0.66 & 0.59 \\
\hline MOZ & 2.68 & 2.59 & & & & & & & & \\
\hline \multicolumn{11}{|l|}{ MRT } \\
\hline MUS & 2.26 & 1.67 & -1.84 & -1.67 & -0.54 & -0.39 & -0.40 & -0.34 & 0.16 & 0.18 \\
\hline \multicolumn{11}{|l|}{ MWI } \\
\hline MYS & 1.76 & 1.51 & -2.37 & -2.19 & -0.87 & -0.73 & -0.81 & -0.72 & -0.16 & -0.11 \\
\hline NAM & 2.49 & 2.44 & 0.27 & 0.79 & 1.07 & 1.43 & 1.03 & 1.36 & 1.45 & 1.70 \\
\hline NER & 2.95 & 2.92 & & & & & & & & \\
\hline NGA & 2.49 & 2.49 & & & & & & & & \\
\hline NIC & 2.63 & 2.62 & -1.80 & -1.56 & -0.37 & -0.20 & -0.12 & 0.01 & 0.50 & 0.61 \\
\hline NLD & -1.30 & -1.31 & -2.15 & -1.98 & 1.49 & 1.90 & -1.52 & -1.40 & -0.35 & -0.20 \\
\hline NOR & -2.88 & -2.64 & -2.21 & -2.16 & -0.31 & 0.01 & -2.05 & -2.10 & -1.25 & -1.24 \\
\hline \multicolumn{11}{|l|}{ NPL } \\
\hline NZL & -1.09 & -0.88 & -2.22 & -1.84 & -2.09 & -2.85 & -1.18 & -0.95 & -0.13 & 0.71 \\
\hline OMN & 1.58 & 1.28 & & & & & & & & \\
\hline PAK & 3.10 & 2.92 & -1.73 & -1.73 & -0.35 & -0.33 & 0.01 & 0.01 & 0.58 & 0.58 \\
\hline PAN & 1.33 & 1.27 & -1.41 & -1.81 & -0.12 & -0.43 & -0.15 & -0.47 & 0.40 & 0.12 \\
\hline PER & 1.78 & 1.50 & -1.77 & -1.90 & -0.32 & -0.40 & -0.26 & -0.38 & 0.37 & 0.25 \\
\hline PHL & 2.14 & 2.16 & -1.31 & -1.42 & 0.03 & -0.05 & 0.13 & 0.06 & 0.72 & 0.67 \\
\hline \multicolumn{11}{|l|}{ PLW } \\
\hline \multicolumn{11}{|l|}{ PNG } \\
\hline POL & 0.47 & 0.22 & -0.99 & -1.62 & -0.10 & -0.40 & -0.07 & -0.50 & 0.14 & -0.24 \\
\hline PRT & 0.19 & 0.10 & -2.03 & -1.34 & -0.81 & -0.68 & -0.91 & -0.51 & -0.66 & -0.60 \\
\hline PRY & 2.17 & 1.95 & -1.67 & -1.73 & -0.26 & -0.27 & -0.18 & -0.22 & 0.48 & 0.45 \\
\hline QAT & -2.15 & -1.47 & -3.03 & -3.07 & 0.31 & 2.61 & -3.19 & -3.03 & -1.88 & -1.33 \\
\hline ROU & 1.39 & 0.87 & -1.95 & -1.79 & -0.47 & -0.40 & -0.42 & -0.46 & 0.14 & 0.02 \\
\hline RUS & 0.20 & -0.11 & -1.83 & -1.71 & -0.17 & -0.41 & -0.48 & -0.60 & 0.00 & -0.54 \\
\hline RWA & 2.73 & 2.71 & & & & & & & & \\
\hline SAU & 0.89 & 0.47 & & & & & & & & \\
\hline \multicolumn{11}{|l|}{ SDN } \\
\hline SEN & 2.90 & 2.81 & -1.11 & -1.01 & 0.14 & 0.22 & 0.31 & 0.37 & 0.88 & 0.93 \\
\hline \multicolumn{11}{|l|}{ SGP } \\
\hline \multicolumn{11}{|l|}{ SLB } \\
\hline \multicolumn{11}{|l|}{ SLE } \\
\hline SLV & 2.37 & 2.29 & -1.81 & -1.66 & -0.41 & -0.29 & -0.26 & -0.16 & 0.37 & 0.45 \\
\hline SRB & 1.39 & 1.31 & -0.53 & -0.32 & 0.40 & 0.48 & 0.39 & 0.46 & 0.74 & 0.76 \\
\hline \multicolumn{11}{|l|}{ STP } \\
\hline \multicolumn{11}{|l|}{ SUR } \\
\hline SVK & 1.20 & 0.58 & -1.26 & -1.30 & -0.31 & -0.49 & -0.32 & -0.45 & 0.03 & -0.25 \\
\hline SVN & -0.63 & -0.62 & -2.26 & -1.93 & -0.42 & -0.75 & -1.11 & -0.89 & 0.77 & 1.61 \\
\hline SWE & -1.80 & -1.28 & -1.94 & -1.63 & 0.11 & 3.22 & -1.36 & -1.18 & -0.51 & -0.01 \\
\hline \multicolumn{11}{|l|}{ SWZ } \\
\hline \multicolumn{11}{|l|}{ SYC } \\
\hline SYR & 2.77 & 2.60 & & & & & & & & \\
\hline \multicolumn{11}{|l|}{ TCD } \\
\hline TGO & 2.70 & 2.57 & & & & & & & & \\
\hline THA & 1.48 & 1.39 & -2.36 & -2.31 & -0.64 & -0.59 & -0.69 & -0.66 & 0.09 & 0.10 \\
\hline & & & & & 23 & & & & & \\
\hline
\end{tabular}




$\begin{array}{ccccccccccc}\text { TJK } & 2.54 & 2.50 & -1.09 & -0.96 & 0.19 & 0.28 & 0.37 & 0.43 & 0.90 & 0.94 \\ \text { TKM } & & & & & & & & & & \\ \text { TLS } & 2.47 & 2.51 & & & & & & & & \\ \text { TON } & 3.18 & 3.09 & & & & & & & & \\ \text { TTO } & 1.62 & 1.51 & & & & & & & & \\ \text { TUN } & 2.22 & 2.02 & -1.30 & -1.02 & -0.08 & 0.11 & 0.01 & 0.16 & 0.54 & 0.64 \\ \text { TUR } & 1.53 & 0.97 & -1.45 & -1.25 & -0.25 & -0.17 & -0.23 & -0.18 & 0.26 & 0.20 \\ \text { TZA } & 2.76 & 2.73 & -1.54 & -1.57 & -0.15 & -0.18 & 0.11 & 0.09 & 0.71 & 0.68 \\ \text { UGA } & 2.72 & 2.68 & -1.62 & -1.44 & -0.21 & -0.07 & 0.03 & 0.13 & 0.66 & 0.75 \\ \text { UKR } & 0.78 & 0.67 & -1.69 & -1.50 & 0.00 & 0.12 & -0.10 & 0.00 & 0.46 & 0.52 \\ \text { URY } & 1.20 & 0.70 & -1.24 & -1.68 & -0.03 & -0.25 & -0.05 & -0.37 & 0.43 & 0.14 \\ \text { USA } & -2.74 & -2.48 & -2.02 & -1.48 & -0.11 & 0.76 & -1.69 & -1.25 & -0.70 & -0.16 \\ \text { UZB } & & & & & & & & & & \\ \text { VCT } & & & & & & & & & & \end{array}$




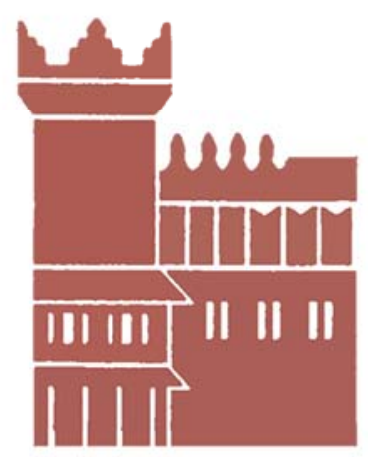

Alma Mater Studiorum - Università di Bologna DEPARTMENT OF ECONOMICS

Strada Maggiore 45

40125 Bologna - Italy

Tel. +39051 2092604

Fax +390512092664

http://www.dse.unibo.it 